\title{
Variación estacional de la comunidad de copépodos en el sector oriental del golfo de Cariaco, estado Sucre, Venezuela
}

\section{Seasonal variation of the community of copepods in the eastern sector of the Gulf of Cariaco, Sucre state, Venezuela}

\author{
Brightdoom Márquez-Rojas ${ }^{2}$, Luis Tróccoli ${ }^{2,3}$ y Evelyn Zoppi de Roa $\dagger^{4}$ \\ $\begin{array}{ll}\text { (iD) } 0000-0002-2703-8696 & \text { (iD) } 0000-0001-8684-6741\end{array}$ \\ 1. Lab. Zooplancton. Dpto. Biología Marina. Instituto Oceanográfico de Venezuela, Universidad de Oriente, Sucre, Venezuela. bmarquez2001@gmail. \\ com *Autora para correspondencia. \\ 2. Instituto de Investigaciones Científicas, Facultad de Ciencias del Mar. Universidad de Oriente, Núcleo Nueva Esparta, Cumaná, Sucre, Venezuela. luis. \\ troccoli@gmail.com \\ 3. Facultad de Ciencias del Mar, Universidad Estatal Península de Santa Elena, Ecuador. \\ 4. Lab. Plancton. Instituto de Zoología y Ecología Tropical, Facultad de Ciencias, Universidad Central de Venezuela, Caracas.evelyn.zoppi@gmail.com
}

\section{RESUMEN}

$\mathrm{L}$ os copépodos son el grupo más numeroso del zooplancton en los sistemas costeros tropicales; ellos se ven afectados principalmente por los gradientes de salinidad, como ocurre en el sector oriental del golfo de Cariaco. El propósito de este estudio fue estudiar las variaciones temporales y espaciales de la estructura de la comunidad de copépodos durante junio, julio, agosto y septiembre de 2009 (época de lluvia) y octubre, noviembre y diciembre de 2009 y enero de 2010 (época de sequía). Las muestras se recolectaron con una red estándar de zooplancton de $60 \mathrm{~cm}$ de diámetro con una malla de $330 \mu \mathrm{m}$ a nivel superficial. La temperatura, salinidad y oxígeno disuelto se tomaron in situ con una sonda multiparamétrica (YSI). La salinidad presentó una marcada variabilidad mensual, con valores entre $32,5 \pm 1,56$ y $38,5 \pm 0.25$ UPS en las épocas de lluvia y sequía, respectivamente. Se identificó un total de 45 taxones de copépodos y Acartia tonsa fue la especie más representativa durante todo el estudio, seguido por Temora turbinata, Paracalanus quasimodo, Subeucalanus subcrassus y S. subtenuis. El presente estudio no mostró un claro patrón estacional en la abundancia, diversidad y la uniformidad de la comunidad de copépodos y los valores de salinidad; por lo tanto, puede considerarse espacialmente uniforme y estacionalmente homogéneo en relación a estos parámetros.

PALABRAS CLAVE: cambios estacionales, copépodos, golfo de Cariaco, sureste Caribe.

\section{ABSTRACT}

$\mathrm{C}$ opepods are the most numerous group of zooplankton in tropical coastal systems; they are mainly affected by salinity gradients, as occurs in the eastern sector of the Gulf of Cariaco. This study purposed to study the temporal and spatial variations in the structure of the copepod community during June, July, August, and September 2009 (rainy season) and October, November, and December 2009 and January 2010 (dry season). Samples were collected with a standard $60 \mathrm{~cm}$ diameter zooplankton net with a $330 \mu \mathrm{m}$ mesh at the surface level. Temperature, salinity, and dissolved oxygen were taken in situ with a multi-parameter probe (YSI). Salinity presented a marked monthly variability, with values between $32.5 \pm 1.56$ and $38.5 \pm 0.25$ UPS in the rainy and dry seasons, respectively. A total of 45 copepod taxa were identified and Acartia tonsa was the most representative species throughout the study, followed by Temora turbinata, Paracalanus quasimodo, Subeucalanus subcrassus and S. subtenuis. The present study did not show a clear seasonal pattern in the abundance, diversity, and uniformity of the copepod community and the salinity values; therefore, it can be considered spatially uniform and seasonally homogeneous concerning these parameters.

KEYWORDS: seasonal changes, copepods, gulf of Cariaco, south-eastern Caribbean. 


\section{INTRODUCCIÓN}

Dentro del zooplancton, los copépodos pertenecientes a los crustáceos constituyen el grupo predominante. Entre ellos, los pelágicos son los más abundantes en los océanos, debido a tres razones: i) cuerpo en forma de torpedo, antenas sensoriales y el fuerte engranaje del motor muscular para la detección y escape de depredadores, ii) la capacidad de detectar presas de forma remota y capturarlas cuando llegan en una corriente de exploración, o cuando nadan a través de la esfera perceptiva del copépodo y iii) a la búsqueda eficiente de pareja en un ambiente diluido, permitiendo la reproducción sexual con apareamiento real en cada generación, poco común en el plancton; esto brinda ventajas en términos de eliminación de mutaciones malas y promoción de "genes buenos" a través de la selección sexual (Kiørboe, 2011). Debido a esto, los copépodos representan entre 60 y $90 \%$ de la biomasa en ambientes costeros (Bradford-Grieve et al., 1999; Ruíz-Pineda et al., 2016; Neumann-Leitão et al., 2018).

El zooplancton presenta variaciones detectables, producto de cambios cíclicos en los factores ambientales (Mclusky y Elliot, 2004; López-Peralta y Mojica-López, 2015; Rose et al., 2019); por consiguiente, los patrones de variabilidad en composición, abundancia y distribución de los copépodos planctónicos, se establecen en función de su acoplamiento con los procesos físicos (i.e.: gradientes de salinidad, temperatura, concentración de nutrientes y patrones internos de circulación) a distintas escalas (Marques et al., 2006; Escamilla et al., 2011; Ruíz-Pineda et al., 2016).

Por otra parte, Magalháes et al. $(2009,2015)$ y Neumann-Leitão et al. (2018) mencionan que la variabilidad estacional y espacial observada en la ocurrencia y distribución de los copépodos planctónicos en sistemas estuarino-costeros tropicales está directamente relacionada con factores abióticos (i.e.: salinidad, turbidezy temperatura) y factores bióticos (i.e.: competencia, depredación, hábitos, disponibilidad y calidad del alimento) o una combinación de ambos. En consecuencia, no se pueden considerar como un conjunto homogéneo de organismos, ya que su distribución no solo cambiará en el espacio y el tiempo, debido a la influencia de factores físicoquímicos, sino también por procesos biológicos (Seda y Devetter, 2000; Beisner, 2001; Vukanic et al., 2018).

En las zonas costeras o estuarinas, los valores de biomasa y diversidad (9 a $50 \mathrm{mg} \mathrm{m}^{-3}$ y 8-10 especies, respectivamente) de los copépodos, son relativamente bajos cuando se comparan con las zonas oceánicas. Estas diferencias se atribuyen a las peculiares condiciones fisiográficas

\section{INTRODUCTION}

Within zooplankton, copepods belonging to crustaceans constitute the predominant group. Among them, pelagics are the most abundant in the oceans, due to three reasons: i) torpedo-shaped body, sensory antennas and the strong gear of the muscular motor for the detection and escape of predators, ii) the ability to detect prey remotely and capture them when they arrive in a current of exploration, or when they swim through the copepod's perceptual sphere and iii) to the efficient search for a partner in a diluted environment, allowing sexual reproduction with real mating in each generation, little common in plankton; This provides advantages in terms of eliminating bad mutations and promoting "good genes" through sexual selection (Kiørboe, 2011). Due to this, copepods represent between 60 and $90 \%$ of the biomass in coastal environments (Bradford-Grieve et al., 1999; Ruíz-Pineda et al., 2016; Neumann-Leitão et al., 2018).

Zooplankton presents detectable variations, a product of cyclical changes in environmental factors (Mclusky and Elliot, 2004; López-Peralta and MojicaLópez, 2015; Rose et al., 2019); therefore, the patterns of variability in composition, abundance, and distribution of planktonic copepods are established based on their coupling with physical processes (i.e.: gradients of salinity, temperature, concentration of nutrients, and internal circulation patterns) at different scales (Marques et al., 2006; Escamilla et al., 2011; Ruíz-Pineda et al., 2016).

On the other hand, Magalháes et al. (2009, 2015) and Neumann-Leitão et al. (2018) mention that the seasonal and spatial variability observed in the occurrence and distribution of planktonic copepods in tropical coastalestuarine systems is directly related to abiotic factors (i.e.: salinity, turbidity, and temperature) and biotic factors (i.e.: competition, predation, habits, availability, and quality of food) or a combination of both. Consequently, they cannot be considered as a homogeneous set of organisms, since their distribution will change not only in space and time, due to the influence of physical-chemical factors, but also due to biological processes (Seda and Devetter, 2000; Beisner, 2001; Vukanic et al., 2018).

In the coastal or estuarine zones, the biomass and diversity values (9 to $50 \mathrm{mg} \mathrm{m}^{-3}$ and $8-10$ species, respectively) of the copepods, are relatively low when compared to the oceanic zones. These differences are attributed to the peculiar physiographic conditions of these areas, which favor a small 
de dichas zonas, que favorecen a un reducido grupo de especies de copépodos (Vásquez-Yeomans et al., 2012; Rice et al., 2015; Ruíz-Pineda et al., 2016). En ese sentido, los gradientes de salinidad resultantes de las interacciones entre la influencia marina y la escorrentía de agua dulce, a menudo representan el parámetro principal para definir los patrones de distribución local y de sucesión de especies (Escamilla et al., 2011; Ruíz-Pineda et al., 2016). El sector oriental del golfo de Cariaco tiene una marcada influencia de agua dulce, debido a los ríos Carinicuao y Cariaco, los cuales mantienen una comunicación con el golfo durante algunos meses del año, originando una zona estuarina (Caraballo, 1982). Por otra parte, los procesos de convección y afloramiento en el golfo de Cariaco tienden a desarrollar fenómenos de renovación de aguas superficiales cálidas por aguas profundas más frías y ricas en nutrientes que estimulan el desarrollo del fitoplancton y del zooplancton (Bonilla et al., 1985). Por lo tanto, el estudio de los patrones de variabilidad de los copépodos en el zooplancton estuarino podría revelar información importante de la dinámica de la comunidad local, con implicaciones a otros sistemas estuarino-costeros tropicales. En ese sentido, el monitoreo de la comunidad de copépodos permite detectar y dar seguimiento a niveles de perturbación y deterioro ecológico (Branco et al., 2007; Escamilla et al., 2011).

Es importante mencionar, que debido a todo lo antes expuesto en el golfo de Cariaco los copépodos han sido ampliamente estudiados habiéndose identificado un número importante de especies. De las 723 especies señaladas para la zona de Venezuela, en dicho golfo se han registrado 136 (Legaré, 1961; Zoppi, 1961; Infante y Urosa, 1986; Márquez-Rojas et al., 2006, 2011, 2014a, 2014b; Razouls et al., 2005-2019). Sin embargo, en el sector oriental o saco del golfo de Cariaco, el conocimiento sobre las comunidades de copépodos es relativamente escaso, a pesar de que varios estudios confirman un marcado gradiente de salinidad en esta zona (Márquez et al., 2011; Martínez et al., 2011).

Actualmente no existe información publicada sobre la estructura de la comunidad de copépodos en el sector oriental del golfo de Cariaco y cómo éstos se ven afectados por las variaciones estacionales en la salinidad, las precipitaciones y la escorrentía de agua dulce. Por consiguiente, el objetivo principal del presente estudio es evaluar la composición taxonómica del ensamble de los copépodos en el sector oriental del golfo de Cariaco y caracterizar los patrones de abundancia y dominancia estacionales en relación con las variaciones anuales de salinidad. group of copepod species (Vásquez-Yeomans et al., 2012; Rice et al., 2015; Ruíz-Pineda et al., 2016). In this sense, the salinity gradients resulting from the interactions between marine influence and freshwater runoff, often represent the main parameter to define the patterns of local distribution and species succession (Escamilla et al., 2011; Ruíz-Pineda et al., 2016). The eastern sector of the Gulf of Cariaco has a marked influence of freshwater, due to the Carinicuao and Cariaco rivers, which maintain communication with the gulf during some months of the year, originating an estuarine area (Caraballo, 1982). On the other hand, convection and outcrop processes in the Gulf of Cariaco tend to develop phenomena of renewal of warm surface waters by colder, nutrient-rich deep waters that stimulate the development of phytoplankton and zooplankton (Bonilla et al., 1985). Therefore, studying the variability patterns of copepods in estuarine zooplankton could reveal important information on the dynamics of the local community, with implications for other tropical estuarine-coastal systems. In this sense, the monitoring of the copepod community allows detecting and following up on levels of ecological disturbance and deterioration (Branco et al., 2007; Escamilla et al., 2011).

It is important to mention that due to all the aforementioned in the Gulf of Cariaco, copepods have been extensively studied, identifying a significant number of species. Of the 723 species indicated for the zone of Venezuela, 136 have been registered in that gulf (Legaré, 1961; Zoppi, 1961; Infante and Urosa, 1986; Márquez-Rojas et al., 2006, 2011, 2014a, 2014b; Razouls et al., 2005-2019). However, in the eastern sector or sac of the Gulf of Cariaco, knowledge about the copepod communities is relatively scarce, although several studies confirm a marked gradient of salinity in this area (Márquez et al., 2011; Martínez et al., 2011).

There is currently no published information on the structure of the copepod community in the eastern sector of the Gulf of Cariaco and how these are affected by seasonal variations in salinity, rainfall, and freshwater runoff. Therefore, the main objective of the present study is to evaluate the taxonomic composition of the copepod assembly in the eastern sector of the Gulf of Cariaco and to characterize patterns of seasonal abundance and dominance to annual variations in salinity. 


\section{MATERIALES Y MÉTODOS}

\section{Área de estudio}

El sector oriental o saco del golfo de Cariaco (Figura 1), tiene aproximadamente $9 \mathrm{~km}$ de ancho y $17 \mathrm{~km} \mathrm{de}$ largo. En este sector se localizan las menores profundidades del golfo, las cuales se aproximan a los 40 metros. En su extremo oriental, desembocan los ríos Carinicuao y Cariaco y en el sur las quebradas Oricoto y López, conformando los mayores afluentes continentales y probablemente la fuente proveedora de gran parte de los materiales sedimentarios que se depositan en el área de estudio (Caraballo, 1982). La vegetación en este sector está caracterizada por la presencia de manglares y zonas xerófilas.

El área de estudio presenta estacionalidad pronunciada que se puede observar en los parámetros físicos de la columna de agua en el comienzo del año causado por el desplazamiento estacional de la zona de convergencia intertropical (ITCZ) que genera una variabilidad significativa en el régimen del viento, las corrientes y descarga de ríos (Lorenzoni et al., 2013; López-Monroy y Tróccoli-Ghinaglia, 2015).

\section{MATERIALS AND METHODS}

\section{Study area}

The eastern sector or sac of the Gulf of Cariaco (Figure 1), is approximately $9 \mathrm{~km}$ wide and $17 \mathrm{~km}$ long. The shallowest depths of the gulf are located in this sector, which is close to 40 meters. At its eastern end, the Carinicuao and Cariaco rivers flow out and in the south, the Oricoto and López ravines, making up the largest continental tributaries and probably the source of supply of much of the sedimentary materials deposited in the study area (Caraballo, 1982). The vegetation in this sector is characterized by the presence of mangroves and xerophytic zones.

The study area presents pronounced seasonality that can be observed in the physical parameters of the water column at the beginning of the year caused by the seasonal displacement of the intertropical convergence zone (ITCZ) that generates significant variability in the wind regime, river currents and discharge (Lorenzoni et al., 2013; LópezMonroy and Tróccoli-Ghinaglia, 2015).

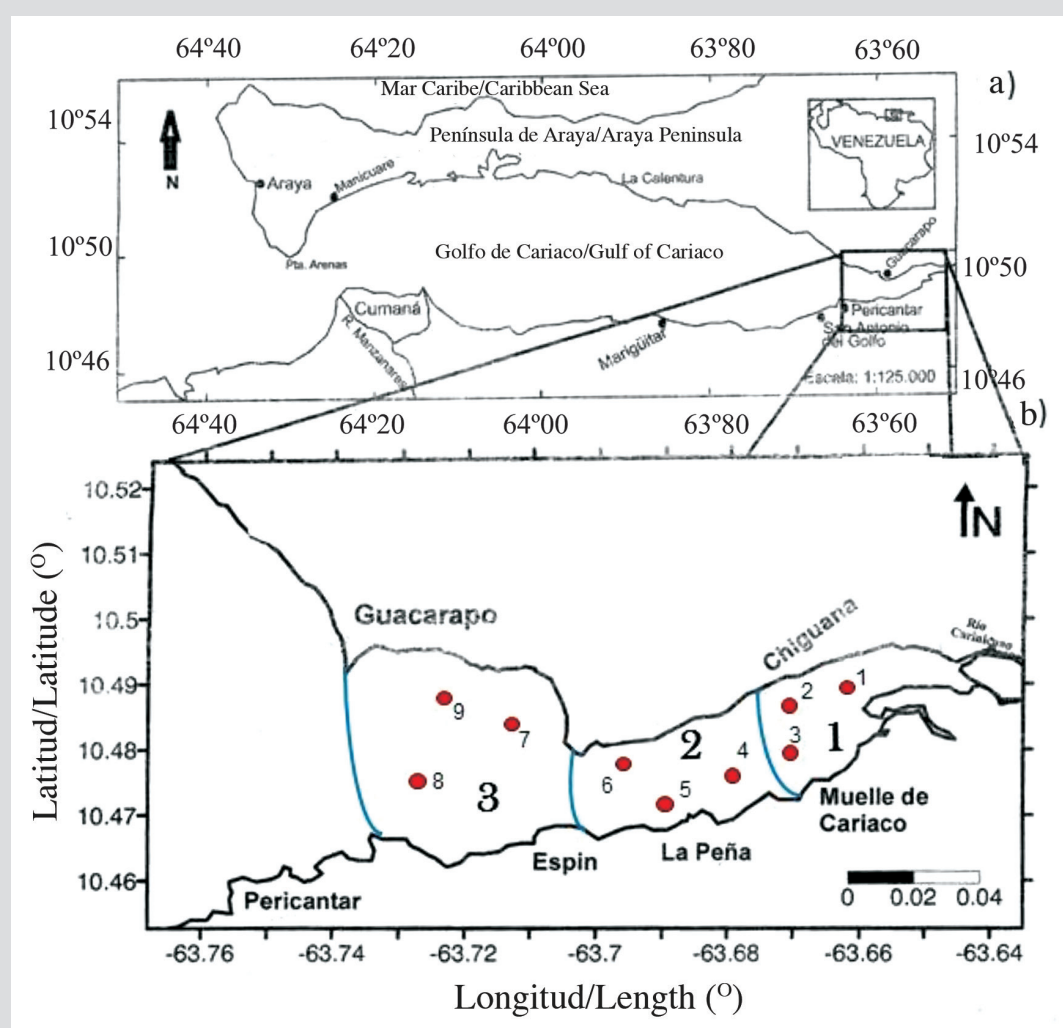

Figura 1. a) Localización geográfica del golfo de Cariaco y b) detalles del extremo oriental o saco del golfo, mostrando las zonas y las estaciones de muestreo.

Figure 1. a) Geographical location of the Gulf of Cariaco and b) details of the eastern end or sac of the gulf, showing the sampling areas and stations. 


\section{Muestreo de campo}

El diseño para tomar las muestras se realizó por medio de un muestreo por conglomerado bietápico con submuestra (Cochran, 1977), el cual consistió en dividir el sector oriental o saco del golfo en tres zonas; dentro de cada zona se escogieron tres estaciones al azar, para un total de 9 muestras por salida (Figura 1b). Las muestras se recolectaron mensualmente entre junio de 2009 y enero de 2010, garantizando la representatividad en el período de lluvias (junio-septiembre) y sequía (octubre-diciembre y enero), en las nueve estaciones en el sector oriental del golfo de Cariaco (Figura 1b).

Las muestras zooplanctónicas fueron recolectadas a nivel superficial, con una red de Bongo (333 y $555 \mu \mathrm{m}$ de abertura de malla y $60 \mathrm{~cm}$ de diámetro), a una velocidad de $3,7 \mathrm{~km} / \mathrm{h}$ durante $15 \mathrm{~min}$. Para el presente trabajo se utilizaron solamente las muestras de mesozooplancton (333 $\mu \mathrm{m}$ ), dicha red estaba equipada con un flujómetro para estimar el volumen de agua filtrado. Todas las muestras fueron inmediatamente colocadas en botellas de poliuretano de $500 \mathrm{~mL}$ de capacidad y conservadas en formalina tamponada con tetraborato sódico, manteniendo la concentración final al $5 \%$.

En cada estación se midieron la temperatura, la salinidad y el oxígeno disuelto superficial por medio de una sonda multiparamétrica (YSI). Simultáneamente, se recolectaron muestras en botellas de polietileno oscuras (2 L) para las determinaciones de clorofila $a$ (Clor. $a$ ) empleando el método espectrofotométrico descrito por Lorenzen modificado por Strickland y Parsons (1972), los resultados se expresaron en mg Clor. $a . \mathrm{m}^{-3}$.

\section{Trabajo en el laboratorio}

Para el zooplancton, cada muestra de la malla de $333 \mu \mathrm{m}$ se homogenizó y dividió en dos porciones iguales por medio de un separador Folsom; una porción fue destinada al estudio de la estructura de la comunidad y la otra para el análisis de la biomasa para otros estudios. Para la determinación taxonómica, de las muestras concentradas (400 mL) se obtuvieron 3 submuestras de $5 \mathrm{~mL}$ con una pipeta Stempel, y se procedió bajo un microscopio estereoscópico, al conteo e identificación de los copépodos adultos y juveniles al más bajo nivel taxonómico posible, empleando las claves de identificación especializadas de Campos-Hernández y Suárez-Morales (1994), SuárezMorales (1995), Bradford-Grieve et al. (1999), así como la base de datos de Razouls et al. (2005-2019). El número de

\section{Field sampling}

The design to take the samples was carried out utilizing a two-stage conglomerate sampling with a subsample (Cochran, 1977), which consisted of dividing the eastern sector or the Gulf sac into three zones; within each zone, three stations were chosen at random, for a total of 9 samples per outlet (Figure 1b). The samples were collected monthly between June 2009 and January 2010, guaranteeing representativeness in the rainy season (JuneSeptember) and drought (October-December and January), in the nine seasons in the eastern sector of the Gulf of Cariaco (Figure 1b).

The zooplankton samples were collected at the surface level, with a Bongo net (333 and $555 \mu \mathrm{m}$ mesh opening and $60 \mathrm{~cm}$ in diameter), at a speed of $3.7 \mathrm{~km} / \mathrm{h}$ for $15 \mathrm{~min}$. For the present work, only the mesozooplankton samples $(333 \mu \mathrm{m})$ were used, this network was equipped with a flowmeter to estimate the volume of filtered water. All samples were immediately placed in $500 \mathrm{~mL}$ polyurethane bottles and stored in formalin buffered with sodium tetraborate, maintaining the final concentration at $5 \%$.

At each station, temperature, salinity, and surface dissolved oxygen were measured using a multi-parameter probe (YSI). Simultaneously, samples were collected in dark polyethylene bottles ( $2 \mathrm{~L}$ ) for determinations of chlorophyll- $a$ (Chlor- $a$ ) using the spectrophotometric method described by Lorenzen modified by Strickland and Parsons (1972), the results were expressed in mg Clor. $-a . \mathrm{m}^{-3}$.

\section{Laboratory work}

For zooplankton, each sample of the $333 \mu \mathrm{m}$ mesh was homogenized and divided into two equal portions utilizing a Folsom separator; one portion was used for the study of community structure and the other for biomass analysis for other studies. For taxonomic determination, from the concentrated samples $(400 \mathrm{~mL}), 3$ sub-samples of $5 \mathrm{~mL}$ were obtained with a Stempel pipette, and a count and identification of adult and juvenile copepods was carried out under the lowest possible taxonomic level, using the specialized identification keys of Campos-Hernández and Suárez-Morales (1994), Suárez-Morales (1995), BradfordGrieve et al. (1999), as well as the Razouls et al. (20052019). The number of organisms counted was divided by the volume of filtered water, so the results were standardized to ind. $\mathrm{m}^{-3}$. The rainfall data analyzed was provided by the Meteorological Service of the National Armed Forces of the Antonio José de Sucre Airport in the city of Cumaná. 
organismos contados se dividió entre el volumen de agua filtrada, por lo que los resultados se estandarizaron a ind. $\mathrm{m}^{-3}$. Los datos de pluviosidad analizados fueron suministrados por el Servicio Meteorológico de la Fuerza Armada Nacional del Aeropuerto Antonio José de Sucre de la ciudad de Cumaná.

\section{Análisis de la información}

Los datos cuantitativos obtenidos de la muestra se usaron para calcular la abundancia absoluta y relativa de las especies de copépodos, mientras los índices ecológicos (diversidad y equidad) se calcularon por mes. La diversidad de los copépodos se calculó usando el índice de ShannonWiener (H') y la equidad a través del índice de Pielou $(J)$ de acuerdo a lo señalado por Krebs (1999).

La comparación de las variables abióticas (temperatura, salinidad y oxígeno disuelto), bióticas (Clor. $a$ ), la abundancia total y diversidad de los copépodos entre las estaciones y zonas se realizó con la prueba no paramétrica Kruskal-Wallis, luego de comprobar que no se cumplieron los supuestos del análisis paramétrico mediante la prueba de normalidad de Kolmogorov-Smirnov y homocedasticidad de Levene (Sheskin, 2004). Las comparaciones de los períodos estudiados, se realizaron con la prueba de Mann-Whitney o test Wilcoxon (Zar, 1999). Se calculó el coeficiente de correlación de Spearman para caracterizar las relaciones entre la salinidad, la abundancia de los copépodos y los índices ecológicos. Para estas pruebas se utilizó el software estadístico Statgraphics plus 5,1.

Para visualizar el comportamiento de la diversidad de los copépodos entre los diferentes períodos estacionales y los meses de muestreo, se realizaron curvas de K-dominancia (Lambshead et al., 1983). En su construcción se utilizan el rango de las especies (en el eje de las abscisas) y el porcentaje de abundancia acumulado (en las ordenadas).

Con los datos de los copépodos se construyeron matrices de disimilitud Bray-Curtis previamente transformados a $\log (\mathrm{x}+1)$, mientras que los datos ambientales (temperatura, oxígeno disuelto, salinidad y clorofila $a$ ) se normalizaron y se emplearon distancias Euclidianas para su construcción (Legendre y Legendre, 1998). Las matrices generadas de los copépodos se utilizaron para analizar la similitud entre las abundancias mensuales por medio de un análisis de clasificación (Cluster) y el escalamiento multidimensional no métrico (nMDS) para visualizar la ordenación de la abundancia de los copépodos en dos dimensiones con respecto a las dos

\section{Analysis of the information}

The quantitative data obtained from the sample were used to calculate the absolute and relative abundance of the copepod species, while the ecological indices (diversity and equity) were calculated per month. Copepod diversity was calculated using the Shannon-Wiener index (H') and equity through the Pielou index $(\mathrm{J})$ according to what Krebs (1999) pointed out.

The comparison of abiotic (temperature, salinity and dissolved oxygen), biotic (Chlor.- $a$ ), total abundance and diversity of copepods between stations and areas were compared with the Kruskal-Wallis non-parametric test, after verifying that the assumptions of the parametric analysis were not met using the Kolmogorov-Smirnov normality test and Levene's homoscedasticity (Sheskin, 2004). The comparisons of the periods studied were made with the Mann-Whitney test or the Wilcoxon test (Zar, 1999). Spearman's correlation coefficient was calculated to characterize the relationships between salinity, copepod abundance, and ecological indices. Statgraphics plus 5.1 statistical software was used for these tests.

To visualize the behavior of the diversity of the copepods between the different seasonal periods and the sampling months, K-dominance curves were performed (Lambshead et al., 1983). In its construction, the range of the species (on the abscissa axis) and the percentage of accumulated abundance (on the ordinate) are used.

Bray-Curtis dissimilarity matrices previously transformed to $\log (x+1)$ were constructed with the copepod data, while the environmental data (temperature, dissolved oxygen, salinity, and chlorophyll-a) were normalized and Euclidean distances were used for their construction. (Legendre and Legendre, 1998). The matrices generated from the copepods were used to analyze the similarity between the monthly abundances utilizing a classification analysis (Cluster) and the non-metric multidimensional scaling (nMDS) to visualize the order of the abundance of the copepods in two dimensions to the two epochs studied; The Primer 6 program was used for these analyzes (Clarke and Gorley, 2006).

To know the possible relationships between the most abundant copepod species and the environmental variables recorded during the two periods studied, a canonical correspondence analysis (ACC) was carried out. This procedure allowed to position the most abundant 
épocas estudiadas; para estos análisis se utilizó el programa Primer 6 (Clarke y Gorley, 2006).

Con el fin de conocer las posibles relaciones entre las especies de copépodos más abundantes y las variables ambientales registradas durante los dos períodos estudiados, se efectuó un análisis de correspondencia canónica (ACC). Este procedimiento permitió posicionar las especies de copépodos más abundantes (>10\%) con las variables temperatura, salinidad, oxígeno disuelto y clorofila $a$, en un sistema sintetizado de coordenadas (componentes 1 y 2) (Ter Braak y Verdonschot, 1995).

\section{RESULTADOS}

\section{Variables abióticas}

En el presente estudio, la temperatura, oxígeno disuelto, salinidad, clorofila $a$, así como la abundancia y los índices ecológicos no mostraron diferencias significativas espaciales $(p>0,05)$ entre las nueve estaciones muestreadas, ni en las tres zonas de estudio $(p>0,05)$. Por lo tanto, los valores reportados para cada mes de muestreo fueron agrupados y evaluados en escalas estacionales (período de lluvia/período de sequía).

La temperatura superficial del agua no mostró una marcada variación entre los períodos estudiados (MannWhitney U-test $=556 ; p>0,05)$, registrando valores entre $24-28,6{ }^{\circ} \mathrm{C}$ en el período de lluvia y $23,4-27,6{ }^{\circ} \mathrm{C}$ para el período de sequía. El oxígeno disuelto mostró marcadas fluctuaciones mensuales, registrando diferencias significativas entre los dos períodos (Mann-Whitney U-test $=156$; $p<0,05)$; en la época de lluvia se registraron los valores más altos $\left(4,9-9,9 \mathrm{mLO}_{2} \cdot \mathrm{L}^{-1}\right)$, mientras en la época de sequía fluctuaron entre $1,4-7,8 \mathrm{mLO}_{2} \cdot \mathrm{L}^{-1}$.

La salinidad presentó una marcada variabilidad mensual, con diferencias de hasta 6 unidades. Se detectaron diferencias significativas estacionales (Mann-Whitney U-test $=1103,5 ; p<0,05)$. El valor más bajo $(32,5 \pm 1,6$; media \pm desviación estándar) fue registrado en junio de 2009 (época de lluvia), mientras el valor más alto $(38,5 \pm 0,2)$ fue medido en enero de 2010 (época de sequía) (Figura 2). Cuando se relaciona la salinidad con las precipitaciones, se observa una tendencia contraria, presentándose los valores más altos de precipitación (17,7 a 73,4 mm) en la época de lluvia (Figura 2). copepod species (>10\%) with the variables temperature, salinity, dissolved oxygen, and chlorophyll- $a$, in a synthesized coordinate system (components 1 and 2) (Ter Braak and Verdonschot, 1995).

\section{RESULTS}

\section{Abiotic variables}

In the present study, temperature, dissolved oxygen, salinity, chlorophyll- $a$, as well as abundance and ecological indices did not show significant spatial differences $(p>0.05)$ between the nine stations sampled, nor in the three study areas $(p>0.05)$. Therefore, the values reported for each month of sampling were grouped and evaluated on seasonal scales (rainy period/dry period).

The surface temperature of the water did not show a marked variation between the periods studied (MannWhitney $\mathrm{U}$-test $=556 ; \mathrm{p}>0.05$ ), registering values between 24-28.6 ${ }^{\circ} \mathrm{C}$ in the rainy period and $23.4-27.6{ }^{\circ} \mathrm{C}$ for the dry period. Dissolved oxygen showed marked monthly fluctuations, registering significant differences between the two periods (Mann-Whitney U-test $=156 ; \mathrm{p}<0.05$ ); the highest values were recorded in the rainy season (4.9-9.9 $\left.\mathrm{mLO}_{2} \cdot \mathrm{L}^{-1}\right)$, while in the dry season they fluctuated between 1.4-7.8 $\mathrm{mLO}_{2} \cdot \mathrm{L}^{-1}$.

Salinity presented marked monthly variability, with differences of up to 6 units. Seasonal significant differences were detected (Mann-Whitney U-test $=1103.5$; $\mathrm{p}<0.05)$. The lowest value $(32.5 \pm 1.6$; mean \pm standard deviation) was recorded in June 2009 (rainy season), while the highest value $(38.5 \pm 0.2)$ was measured in January 2010 (dry season) (Figure 2). When salinity is related to precipitation, a contrary trend is observed, presenting the highest precipitation values (17.7 to $73.4 \mathrm{~mm}$ ) in the rainy season (Figure 2). 


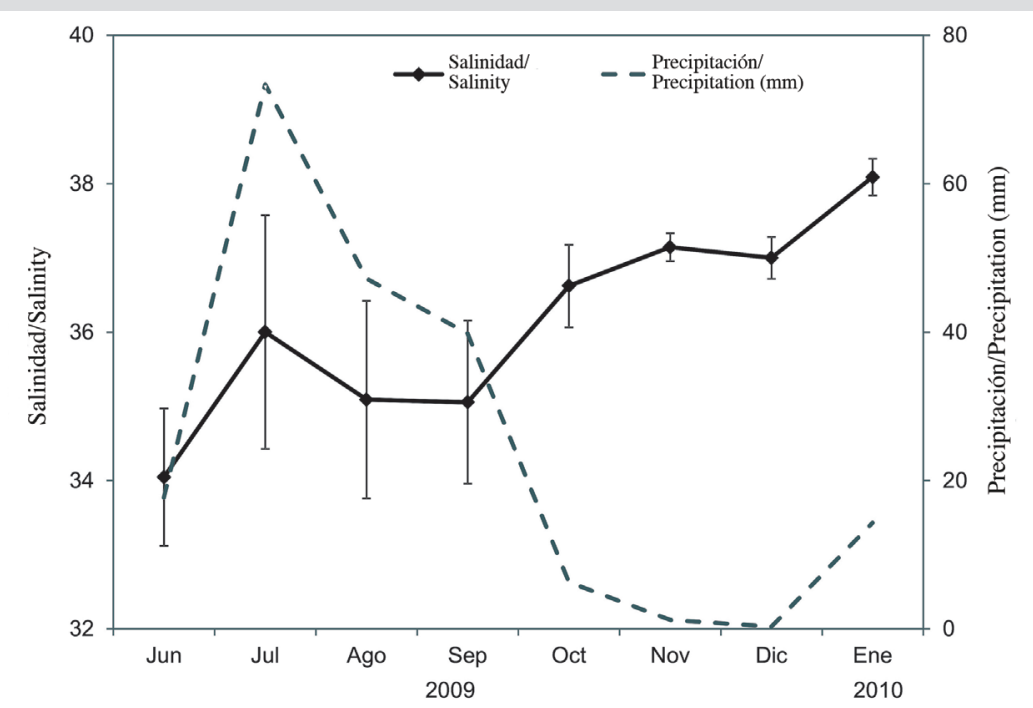

Figura 2. Variación estacional de la salinidad (media \pm desviación estándar) en relación con la precipitación mensual en la ciudad de Cumaná, en el saco del golfo de Cariaco, estado Sucre, Venezuela.
Figure 2. Seasonal variation in salinity (mean \pm standard deviation) about monthly precipitation in the city of Cumaná, in the Gulf of Cariaco sack, Sucre state, Venezuela.

\section{Variables bióticas}

La concentración de Clor. a presentó una gran fluctuación durante el estudio, con valores comprendidos entre no detectable (ND) y $26,5 \mathrm{mg}$ Clor.a.m $\mathrm{m}^{-3}$. Las concentraciones más altas se midieron al comienzo del estudio (época de lluvia), registrando en julio de 2009 magnitudes de 17,6 $\pm 6.4 \mathrm{mg}$ Clor. $a . \mathrm{m}^{-3}$. Durante los meses restantes, los valores medios de Clor. $a$ fueron más bajos, oscilando entre 0,9 y $8,9 \mathrm{mg}$ Clor. $a . \mathrm{m}^{-3}$. Se detectó diferencias significativas (Mann-Whitney U-test $=1008$; $p<0,05)$ entre los períodos estudiados.

\section{Composición y abundancia de copépodos}

Se identificaron un total de 45 especies de copépodos (Tabla 1). La abundancia media mensual de los copépodos varió entre $513 \pm 79$ ind. $\mathrm{m}^{-3}$ en diciembre de 2010 (época de sequía) y $7156 \pm 627$ ind. $\mathrm{m}^{-3}$ en julio de 2009 (época de lluvia) (Figura 3a). Acartia tonsa (Dana) fue la más abundante y representativa de todo el estudio, aportando un $83,2 \pm 2,6 \%$ en lluvia y $60,8 \pm 4,7 \%$ en época de sequía del total de la abundancia de los copépodos (Tabla 1). A escala mensual, la abundancia de esta especie superó el $90 \%$ en julio, noviembre y diciembre de 2009 (Figura 5c). A esta especie le siguió Temora turbinata (Dana), con una abundancia relativa de 5,3 y 30,3\% para los períodos de lluvia y sequía, respectivamente. Durante el período de sequía (octubre de 2009 y enero de 2010), se obtuvieron las abundancias más altas de esta especie. Paracalanus quasimodo (Bowman) fue la tercera especie en

\section{Biotic variables}

Chlorine concentration showed great fluctuation during the study, with values ranging from not detectable (ND) to $26.5 \mathrm{mg}$ Chlor.a. $\mathrm{m}^{-3}$. The highest concentrations were measured at the beginning of the study (rainy season), registering magnitudes of $17.6 \pm 6.4 \mathrm{mg}$ Chlor.a.m ${ }^{-3}$ in July 2009. During the remaining months, the average Chlorine values were lower, ranging from 0.9 to $8.9 \mathrm{mg}$ Chlor.a. $\mathrm{m}^{-3}$. Significant differences (MannWhitney U-test $=1008 ; \mathrm{p}<0.05)$ were detected between the periods studied.

\section{Composition and abundance of copepods}

A total of 45 species of copepods were identified (Table 1 ). The average monthly abundance of copepods varied between $513 \pm 79$ ind. $\mathrm{m}^{-3}$ in December 2010 (dry season) and $7156 \pm 627$ ind. $\mathrm{m}^{-3}$ in July 2009 (rainy season) (Figure 3a). Acartia tonsa (Dana) was the most abundant and representative of the entire study, contributing $83.2 \pm 2.6 \%$ in rain and $60.8 \pm 4.7 \%$ in the dry season of the total abundance of copepods (Table 1). On a monthly scale, the abundance of this species exceeded $90 \%$ in July, November, and December 2009 (Figure 5c). This species was followed by Temora turbinata (Dana), with a relative abundance of 5.3 and $30.3 \%$ for the periods of rain and drought, respectively. During the drought period (October 2009 and January 2010), the highest abundances of this species were obtained. Paracalanus quasimodo (Bowman) was the third species in order of abundance, 
orden de abundancia, sobresaliendo en el período de lluvia (2,15\%), principalmente en junio y septiembre de 2009. excelling in the rainy season (2.15\%), mainly in June and September 2009.
Tabla 1. Composición taxonómica, abundancia absoluta (media \pm DS) y relativa de los copépodos colectados durante la época de lluvia y sequía en el saco del golfo de Cariaco, estado Sucre, Venezuela.
Table 1. Taxonomic composition, absolute (mean \pm SD), and relative abundance of the copepods collected during the rainy and dry season in the Gulf of Cariaco sack, Sucre state, Venezuela.

\begin{tabular}{|c|c|c|c|c|}
\hline Taxones/Taxons & Lluvia/Rain & $\%$ & Sequía/Drought & $\%$ \\
\hline \multicolumn{5}{|c|}{ ORDEN CALANOIDA/CALANOID ORDER } \\
\hline Acartia (Acanthacartia) tonsa (Dana, 1849) & $437.9 \pm 417.6$ & 83.20 & $237.1 \pm 406.2$ & 60.83 \\
\hline A. (Acartia) danae (Giesbrecht, 1889) & & & 3.7 & 0.03 \\
\hline A.(Odontacartia) lilljeborgi (Giesbrecht, 1889) & 3.6 & 0.02 & 1.9 & 0.08 \\
\hline A. (Acartia) negligens (Dana, 1849) & & & 11.3 & 0.01 \\
\hline Euchirella rostrata (Claus, 1866) & & & $3.3 \pm 3.2$ & 0.05 \\
\hline Nannocalanus minor (Claus, 1863) & $22.18 \pm 21.7$ & & & \\
\hline Centropages velificatus (Oliveira, 1947) & $29.0 \pm 36.6$ & 0.61 & $4.08 \pm 4.95$ & 0.20 \\
\hline Clausocalanus furcatus (Brady, 1883) & & & $7.78 \pm 5.70$ & 0.11 \\
\hline C. arcuicornis (Dana, 1849) & & & $16.87 \pm 4.11$ & 0.48 \\
\hline Subeucalanus crassus (Giesbrecht, 1888) & & & $3.22 \pm 3.03$ & 0.04 \\
\hline S. subcrassus (Giesbrecht, 1888) & $10.9 \pm 15.1$ & 0.97 & $11.97 \pm 10.18$ & 0.60 \\
\hline S. subtenuis (Giesbrecht, 1888) & $12.4 \pm 11.3$ & 0.99 & $10.5 \pm 8.62$ & 0.67 \\
\hline Paracalanus aculeatus (Giesbrecht, 1888) & $16.9 \pm 25.1$ & 0.53 & $12.3 \pm 13.58$ & 0.44 \\
\hline P. quasimodo (Bowman, 1971) & $24.9 \pm 34.7$ & 2.15 & $17.4 \pm 19.57$ & 1.49 \\
\hline Labidocera scotti (Giesbrecht, 1897) & $4.35 \pm 0.3$ & 0.05 & & \\
\hline L. detruncata (Dana, 1849) & 1.5 & 0.01 & & \\
\hline Pseudodiaptomus marshi (Wright, 1936) & 1.87 & 0.01 & & \\
\hline P. pelagicus (Herrick, 1884) & 2.7 & 0.01 & 2.14 & 0.02 \\
\hline P. cokeri (Gonzalez\&Bowman, 1965) & 0.7 & & & \\
\hline Temora stylifera (Dana, 1849) & $34.9 \pm 38.9$ & 2.21 & 1.07 & 0.01 \\
\hline T. turbinata (Dana, 1849) & $209.6 \pm 292.9$ & 5.35 & $132.71 \pm 329.2$ & 30.26 \\
\hline \multicolumn{5}{|c|}{ ORDEN CYCLOPOIDA/CYCLOPOID ORDER } \\
\hline Oithona atlantica (Farran, 1908) & 7.5 & 0.04 & 2.15 & 0.01 \\
\hline O. nana (Giesbrecht, 1892) & $4.3 \pm 3.9$ & 0.32 & $1.61 \pm 0.75$ & 0.02 \\
\hline O. plumifera (Baird, 1843) & $1.7 \pm 1.0$ & 0.04 & $17.21 \pm 19.95$ & 1.10 \\
\hline O. setigera (Dana, 1849) & $3.6 \pm 4.8$ & 0.27 & $6.48 \pm 7.50$ & 0.55 \\
\hline O. similis (Claus, 1866) & $3.9 \pm 4.0$ & 0.22 & $2.10 \pm 1.08$ & 0.04 \\
\hline \multicolumn{5}{|c|}{ SUBORDEN POECILOSTOMATOIDA/POECILOSTOMATOIDA SUBORDER } \\
\hline Saphirella tropica (Wolfenden, 1906) & 1.9 & 0.01 & & \\
\hline Agetus flaccus (Giesbrecht, 1891) & $8.32 \pm 6.3$ & 0.13 & 4.45 & 0.03 \\
\hline A.typicus (Krøyer, 1849) & $4.3 \pm 7.2$ & 0.09 & $6.8 \pm 4.84$ & 0.14 \\
\hline A. limbatus (Brady, 1883) & 2.1 & 0.01 & 2.15 & 0.01 \\
\hline Corycaeus clausi (F. Dahl, 1894) & $6.2 \pm 8.7$ & 0.23 & $3.07 \pm 2.72$ & 0.13 \\
\hline C. speciosus (Dana, 1849) & $12.7 \pm 24.7$ & 1.00 & $5.08 \pm 4.69$ & 0.40 \\
\hline Ditrichocorycaeus amazonicus (F. Dahl, 1894) & $17.9 \pm 21.1$ & 0.47 & $37.23 \pm 28.01$ & 0.80 \\
\hline Onychocorycaeus catus (F. Dahl, 1894) & $6.4 \pm 4.6$ & 0.44 & $10.87 \pm 15.7$ & 0.62 \\
\hline O. latus (Dana, 1849) & $7.1 \pm 5.3$ & 0.26 & 1.07 & 0.01 \\
\hline
\end{tabular}




\begin{tabular}{|l|c|c|c|c|}
\hline \multicolumn{1}{|c|}{ Taxones/Taxons } & Lluvia/Rain & $\%$ & Sequía/Drought & \% \\
\hline Urocorycaeus furcifer (Claus, 1863) & $7.1 \pm 3.1$ & 0.07 & $4.29 \pm 4.55$ \\
\hline U. lautus (Dana, 1849) & $8.2 \pm 11.7$ & 0.17 & $2.27 \pm 0.23$ \\
\hline U. longistylis (Dana, 1849) & 6.2 & 0.03 & 0.06 \\
\hline Farranula carinata (Giesbrecht, 1891) & & & $1.48 \pm 0.57$ \\
\hline F. gracilis (Dana, 1849) & $2.33 \pm 2.67$ & 0.01 & 0.02 \\
\hline Oncaea media (Giesbrecht, 1891) & $2.4 \pm 1.7$ & 0.10 & $3.13 \pm 1.97$ \\
\hline O. mediterranea (Claus, 1863) & $2.0 \pm 0.1$ & 0.02 & $4.60 \pm 2.75$ \\
\hline O. notopus (Giesbrecht, 1891) & 0.8 & 0.01 & 0.18 \\
\hline O. venusta (Philippi, 1843) & 10.5 & 0.06 & $5.73 \pm 4.34$ \\
\hline Euterpina acutifrons (Dana, 1848) & ORDEN HARPACTICOIDA/HARPACTICOIDA ORDER & 0.12 \\
\hline
\end{tabular}

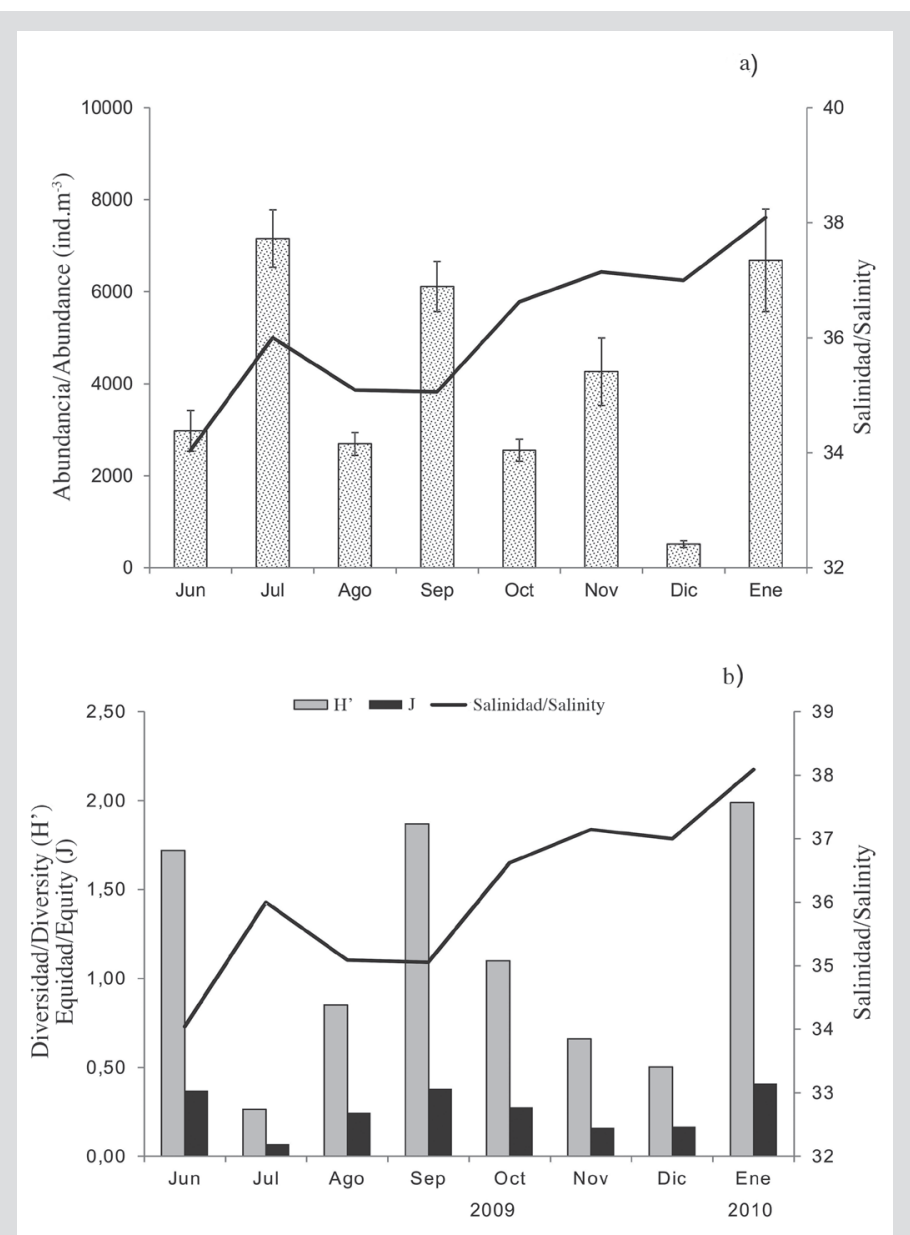

Figura 3. a) Media mensual ( \pm desviación estándar) de la abundancia de los copépodos y b) índices ecológicos de los copépodos recolectados en el saco del golfo de Cariaco, estado Sucre, Venezuela. Estos datos siempre fueron dibujados con la salinidad (línea).
Figure 3. a) Monthly mean ( \pm SD standard deviation) of the abundance of copepods and b) ecological indices of copepods collected in the Gulf of Cariaco sack, Sucre state, Venezuela. These data were always drawn with salinity (line).
Subeucalanus subcrassus y S. subtenuis, siguieron en orden de abundancia, con representaciones relativamente bajas $(0,97$ y $0,99 \%$, respectivamente) y destacándose en el período de lluvia (Tabla 1, Figura 5c).
Subeucalanus subcrassus and S. subtenuis followed in order of abundance, with relatively low representations ( 0.97 and $0.99 \%$, respectively) and standing out in the rainy season (Table 1, Figure 5c). 
Dentro de las principales especies, $A$. tonsa $(\mathrm{K}-\mathrm{W}=28,3, p<0,001)$ y $T$. turbinata $(\mathrm{K}-\mathrm{W}=23,5$, $p<0,001)$ mostraron diferencias significativas estacionales en la abundancia. Variaciones estacionales también fueron observadas en la abundancia total de los copépodos $(\mathrm{K}-\mathrm{W}=31,4, p<0,001)$. El coeficiente de Spearman mostró relaciones significativas entre la salinidad y la abundancia de T. turbinata $(r s=0,37, p<0,05)$, entre la abundancia de los copépodos y $A$. tonsa $(r s=0,72 ; p<0,001)$ y $T$. turbinata ( $r s=0,57 ; p<0,01)$. Por otro lado, se hallaron correlaciones significativas negativas entre la diversidad y equidad con $A$. tonsa ( $r s=-0,57$ y $r s=-0,66 ; p<0,001$, respectivamente) y entre la abundancia y la equidad $(r s=-0,49 ; p<0,01)$.

\section{Estructura comunitaria}

Las fluctuaciones mensuales de la abundancia, diversidad y equidad de los copépodos fueron diagramadas con respecto a la salinidad (Figura 3b). No se detectaron diferencias significativas en la diversidad de los copépodos con respecto a los períodos estudiados (Mann-Whitney $\mathrm{U}$-test $=641 ; p>0,05)$. Los valores fueron similares durante los dos períodos, registrando en la época de lluvia valores medios de 1,2 $\pm 0,8$ bits.ind $^{-1}$, con un mínimo de 0,3 bits.ind ${ }^{-1}$ en julio de 2009 y un máximo de 1.9 bits.ind $^{-1}$ en septiembre de 2009. En la época de sequía se obtuvo un valor medio de $1,1 \pm 0,9$ bits.ind $^{-1}$, con un mínimo de 0.5 bits.ind $^{-1}$ en diciembre de 2009 y un máximo de 2,0 bits.ind ${ }^{-1}$ en enero de 2010. La leve disminución de los valores de diversidad durante los meses de sequía, coincidió con la menor abundancia de copépodos (Figura 3b). Resultados contrarios fueron hallados para la equidad, encontrándose diferencias significativas entre los períodos (Mann-Whitney U-test $=808,5 ; p<0,05)$, pero al igual que en la diversidad, los valores mayores se registraron en lluvia $(0,3 \pm 0,1)$, mientras que en sequía la media fue de $0,2 \pm 0,1$.

La curva de K-dominancia durante la época de lluvia mostró alta dominancia con menor diversidad durante julio y agosto, mientras que junio y septiembre reveló menor dominancia con una mayor diversidad de especies (Figura 4a). Durante los tres primeros meses del período de sequía, tuvieron un comportamiento similar, alta dominancia con menor cantidad de especies, mientras que enero de 2010 registró la menor dominancia del estudio con mayor diversidad de especies (Figura 4b). La curva de K-dominancia también exhibió diferencia en la diversidad y en la abundancia relativa de las especies entre los períodos estudiados (Figura 4c), mostrando en la época de sequía menor dominancia de las especies más abundantes, esto se puede corroborar en la Tabla 1.
Among the main species, A. tonsa $(\mathrm{K}-\mathrm{W}=28.3$, $\mathrm{p}<0.001)$ and T. turbinata $(\mathrm{K}-\mathrm{W}=23.5, \mathrm{p}<0.001)$ showed significant seasonal differences in abundance. Seasonal variations were also observed in the total abundance of copepods $(\mathrm{K}-\mathrm{W}=31.4, \mathrm{p}<0.001)$. Spearman's coefficient showed significant relationships between salinity and abundance of $T$. turbinata ( $\mathrm{rs}=0.37, \mathrm{p}<0.05$ ), between abundance of copepods and $A$. tonsa (rs $=0.72 ; \mathrm{p}<0.001)$ and T. turbinata $(\mathrm{rs}=0.57 ; \mathrm{p}<0.01$ ). On the other hand, significant negative correlations were found between diversity and equity with $A$. tonsa ( $\mathrm{rs}=-0.57$ and $\mathrm{rs}=-0.66$; $\mathrm{p}<0.001$, respectively) and between abundance and equity $(\mathrm{rs}=-0.49 ; \mathrm{p}<0.01)$.

\section{Community structure}

The monthly fluctuations in the abundance, diversity, and equity of the copepods were plotted with respect to salinity (Figure $3 \mathrm{~b}$ ). No significant differences were detected in the diversity of the copepods to the periods studied (Mann-Whitney U-test $=641 ; \mathrm{p}>0.05$ ). The values were similar during the two periods, registering average values of $1.2 \pm 0.8$ bits.ind $^{-1}$ in the rainy season, with a minimum of 0.3 bits.ind $^{-1}$ in July 2009 and a maximum of 1.9 bits.ind $^{-1}$ in September 2009. In the dry season, an average value of $1.1 \pm 0.9$ bits.ind $^{-1}$ was obtained, with a minimum of 0.5 bits.ind $^{-1}$ in December 2009 and a maximum of 2.0 bits.ind $^{-1}$ in January 2010. The slight decrease of the diversity values during the drought months coincided with the lower abundance of copepods (Figure 3b). Contrary results were found for equity, finding significant differences between the periods (Mann-Whitney U-test $=808.5 ; \mathrm{p}<0.05)$, but as in diversity, the highest values were recorded in rain $(0.3 \pm 0.1)$, while in drought the mean was $0.2 \pm 0.1$.

The K-dominance curve during the rainy season showed high dominance with less diversity during July and August, while June and September revealed less dominance with greater species diversity (Figure 4a). During the first three months of the drought period, they had similar behavior, high dominance with fewer species, while January 2010 registered the lowest dominance of the study with greater species diversity (Figure 4b). The K-dominance curve also exhibited a difference in the diversity and relative abundance of the species between the periods studied (Figure $4 \mathrm{c}$ ), showing less dominance of the most abundant species in the dry season, this can be corroborated in Table 1 . 


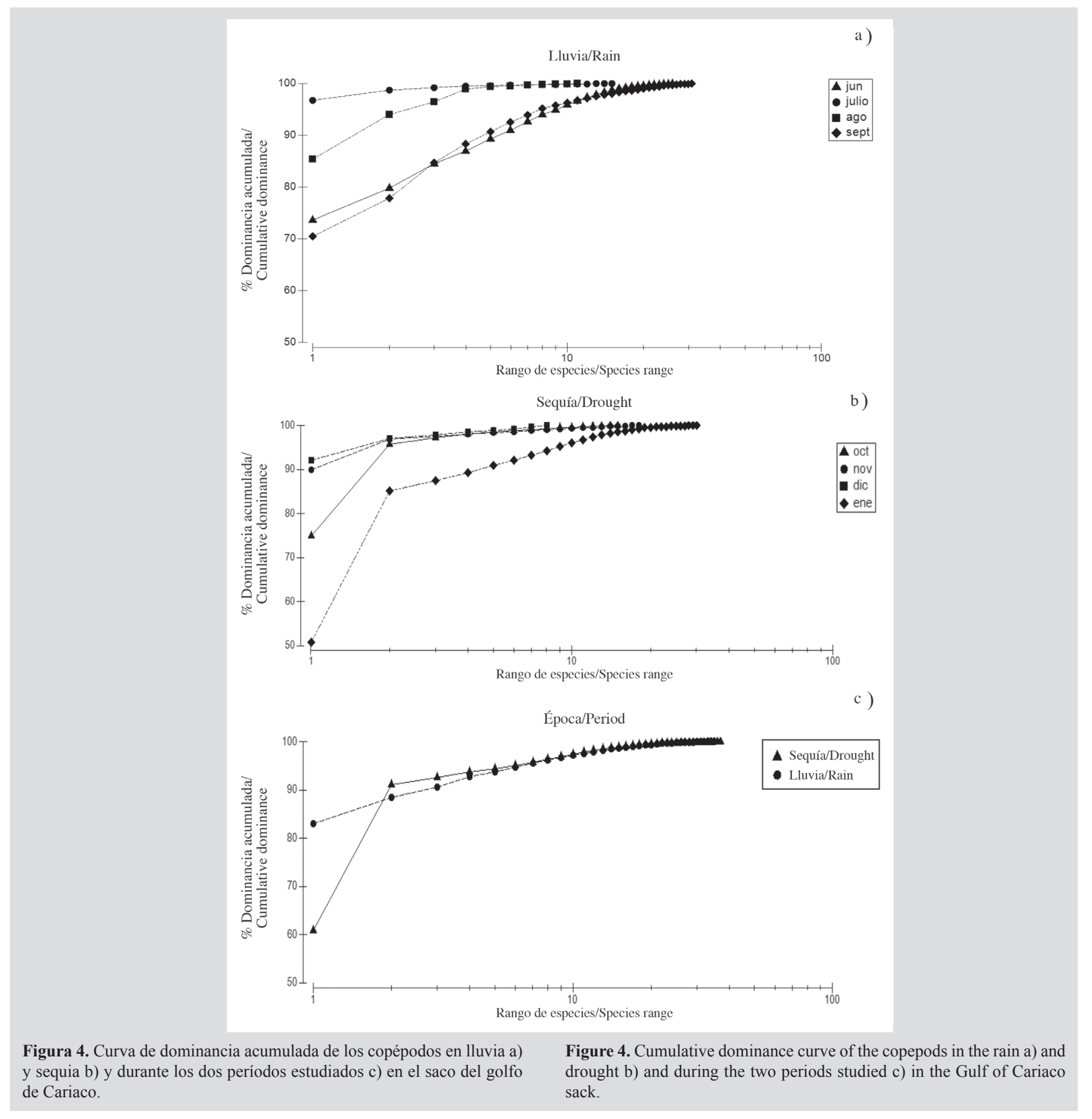

De acuerdo a los análisis de Cluster y nMDS agrupamos a la comunidad de copépodos del saco del golfo de Cariaco en dos conjuntos de acuerdo a la variabilidad estacional (Figura 5a, Figura 5b). En el Cluster, a un corte al $75 \%$ de similitud, se observan estos dos grupos. El primero entre noviembre y diciembre de 2009 (época de sequía). Durante estos meses se observó un elevado porcentaje de abundancia (>80\%) de Acartia tonsa asociada con una salinidad relativamente alta (Figura 5c). El segundo grupo se puede dividir en dos subgrupos, un primer subgrupo en julio,
According to the Cluster and nMDS analyzes, we grouped the copepod community of the Gulf of Cariaco sack into two groups according to seasonal variability (Figure 5a, Figure 5b). In the Cluster, at a cutoff of $75 \%$ similarity, these two groups are observed. The first between November and December 2009 (dry season). During these months a high percentage of abundance (> $80 \%)$ of Acartia tonsa was observed, associated with relatively high salinity (Figure 5c). The second group can be divided into two subgroups, the first subgroup in July, August and 
agosto y octubre de 2009 con porcentajes de abundancia altos (75-95\%) de Acartia tonsa relacionada con alta salinidad y el segundo subgrupo en junio y septiembre de 2009 y enero de 2010 con una mejor distribución de los porcentajes de abundancias entre las otras especies de copépodos Temora turbinata y Paracalanus quasimodo (Figura 5c). En el nMDS, el grupo I, lo constituyeron principalmente los meses de lluvia, donde T. turbinata y P. quasimodo registraron sus mayores porcentajes de abundancia; no obstante, el grupo II se asoció con la época de sequía, donde el porcentaje de abundancia de $A$. tonsa fue casi absoluto (Figura $5 b$ ).
October 2009 with high abundance percentages (75-95\%) of Acartia tonsa relating to high salinity and the second subgroup in June and September 2009 and January 2010 with a better distribution of the percentages of abundances among the other copepod species Temora turbinata and Paracalanus quasimodo (Figure 5c). In nMDS, group I mainly consisted of the rainy months, where $T$. turbinata and P. quasimodo registered their highest percentages of abundance; however, group II was associated with the dry season, when the percentage of the abundance of $A$. tonsa was almost absolute (Figure 5b).

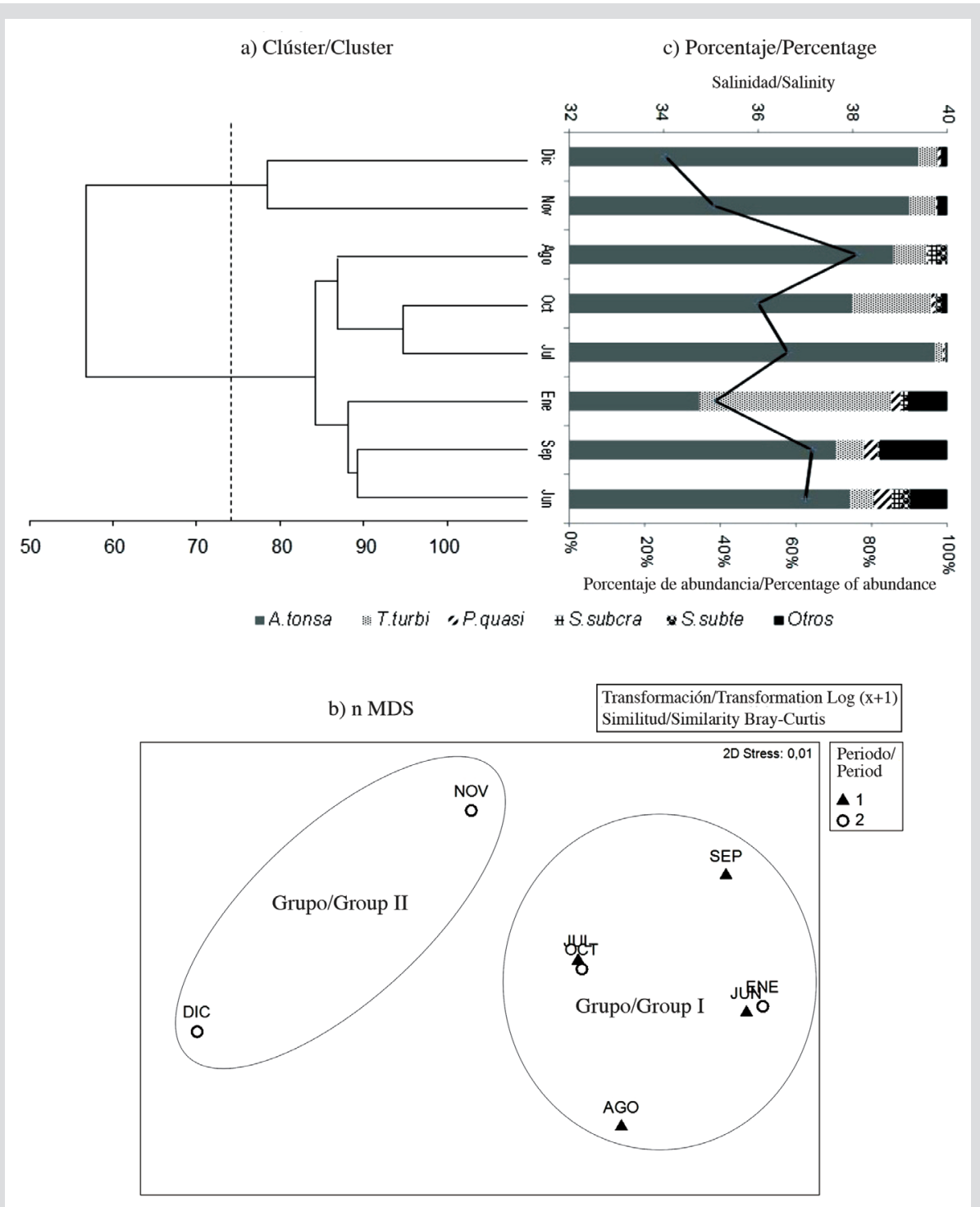

Figura 5. a) Análisis de Cluster y b) nMDS basado en la abundancia de los taxa de copépodos. c) Porcentaje de abundancia de los taxa de copépodos con la salinidad (línea continua negra) muestreados en el saco del golfo de Cariaco. A.tonsa: Acartia tonsa, T.turbi: Temora turbinata, P.quasi: Paracalanus quasimodo, S.subcra: Subeucalanus subcrassus, S.subte: Subeucalanus subtenuis. Período lluvia (triángulo negro) y sequía (círculo blanco).
Figure 5. a) Cluster analysis and b) nMDS based on the abundance of the copepod taxa. c) Percentage of the abundance of copepod taxa with salinity (solid black line) sampled in the Gulf of Cariaco sack. A.tonsa: Acartia tonsa, T. turbi: Temora turbinata, P.quasi: Paracalanus quasimodo, S.subcra: Subeucalanus subcrassus, S.subte: Subeucalanus subtenuis. Rain period (black triangle) and drought (white circle). 
El ACC considerando las especies más abundantes, durante la época de lluvia, mostró una correlación entre variables ambientales y el conjunto de especies de 0,69 , se caracterizó por una relación directa de A. tonsa con la salinidad y la clorofila $a$, mientras que el componente 2 , que tuvo una correlación ambiente-especies de 0,45 , estuvo caracterizado por una relación directa de $T$. turbinata y $P$. quasimodo con la temperatura y el oxígeno disuelto (Figura 6a).

Por otra parte, en la época de sequía, mostró una correlación ambiente-especies de 0,69 , se caracterizó por una relación directa de $A$. tonsa con la salinidad y el oxígeno disuelto, mientras que el componente 2, tuvo una correlación ambiente-especies de 0,43 , estuvo caracterizado por una relación directa de $S$. subcrassus con la salinidad y la clorofila $a$ con $P$. aculeatus. Los resultados del ACC corroboran lo encontrado en las correlaciones no paramétricas de Spearman, que sugiere que la abundancia de las especies más abundantes de copépodos mostraron variaciones estacionales significativas, principalmente en A. tonsa y T. turbinata e incluso en estas especies quedó demostrado las relaciones significativas con la salinidad.
The ACC considering the most abundant species, during the rainy season, showed a correlation between environmental variables and the set of species of 0.69 , it was characterized by a direct relationship of $A$. tonsa with salinity and chlorophyll- $a$, while the component 2 , which had an environment-species correlation of 0.45 , was characterized by a direct relationship between $T$. turbinata and $P$. quasimodo with temperature and dissolved oxygen (Figure 6a).

On the other hand, in the dry season, it showed an environment-species correlation of 0.69 , it was characterized by a direct relationship of $A$. tonsa with salinity and dissolved oxygen, while component 2 , had an environment-species correlation of 0.43 , was characterized by a direct relationship of $S$. subcrassus with salinity and chlorophyll- $a$ with $P$. aculeatus. The ACC results corroborate what was found in Spearman's non-parametric correlations, which suggests that the abundance of the most abundant species of copepods showed significant seasonal variations, mainly in A. tonsa and T. turbinata and even in these species the relationships were demonstrated significant with salinity.

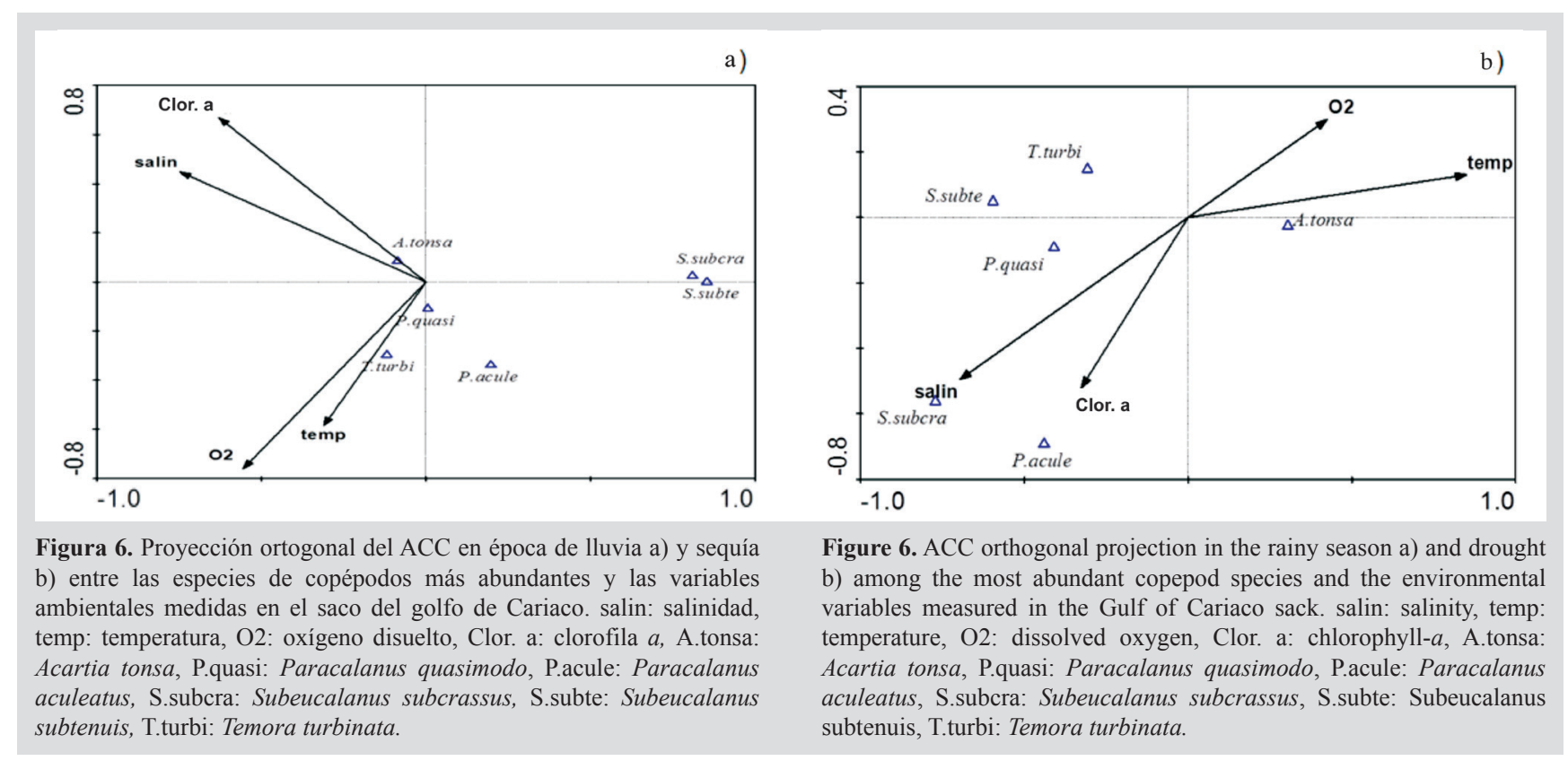




\section{DISCUSIÓN}

Al considerar los ecosistemas estuarinos tropicales en Venezuela (Zoppi, 1974; Gómez, 1983; Briceño et al., 2009; Márquez-Rojas et al., 2017; Villalba et al., 2017) y en todo el mundo (Sterza y Fernandes, 2006; Garboza da Costa et al., 2008; Magalháes et al., 2009; Da Costa et al., 2011) se corrobora que los cambios estacionales en la salinidad están influenciados principalmente por las lluvias. Esta afirmación quedo corroborada en el saco del golfo de Cariaco con la marcada fluctuación estacional que presentó la salinidad. Los valores elevados en las precipitaciones registrados entre junio y septiembre de 2010, incrementaron los caudales de los ríos, especialmente Carinicuao y Cariaco, reflejando una disminución de los valores de salinidad en la época de lluvias. Esta variación temporal de la salinidad en el extremo oriental del golfo coincidió con lo mencionado por Simpson y Griffiths (1967), Okuda et al. (1978) y más recientemente con Márquez et al. (2011), Martínez et al. (2011), MárquezRojas et al. (2017).

El patrón estacional de distribución observado en la abundancia total de copépodos, con los valores más altos registrados en la época de lluvias, estuvo en parte relacionada con el comportamiento de Acartia tonsa, que fue la especie más abundante; esto también fue encontrado en muchos otros sistemas estuarinos (Magalháes et al., 2009; Da Costa et al., 2011; Escamilla et al., 2011; Teiguel-C, 2015; RuízPineda et al., 2016). Este copépodo calanoide planctónico es una especie cosmopolita, dominante en muchas zonas subtropicales y zonas templadas (Mauchline, 1998), muestra alto grado de tolerancia a los cambios ambientales logrando soportar normalmente intervalos de salinidad desde 5 UPS (Cervetto et al., 1999) hasta 30 UPS (Calliari et al., 2006), excepcionalmente se han encontrado ejemplares en sistemas con salinidad de 0 UPS (Cronin et al., 1962) hasta 52 UPS (Rey et al., 1991).

La ausencia de correlación significativa entre Acartia tonsa y la salinidad en el saco del golfo de Cariaco, indica que esta especie no demostró preferencia por las aguas estuarinas. Sin embargo, la tolerancia intraespecífica a las salinidades bajas y moderadas podría explicar la mayor cantidad numérica de $A$. tonsa durante todo el período de lluvia. Para Temora turbinata, una especie ampliamente distribuida en regiones tropicales y subtropicales, miembro conspicuo de las comunidades mesozooplanctónicas superficiales y cercanas a la superficie en estuarios y en aguas neríticas y oceánicas (Ara, 2002), la correlación entre

\section{DISCUSSION}

When considering tropical estuarine ecosystems in Venezuela (Zoppi, 1974; Gómez, 1983; Briceño et al., 2009; Márquez-Rojas et al., 2017; Villalba et al., 2017) and throughout the world (Sterza and Fernandes, 2006; Garboza da Costa et al., 2008; Magalháes et al., 2009; Da Costa et al., 2011) it is corroborated that seasonal changes in salinity are mainly influenced by rainfall. This statement was corroborated in the Gulf of Cariaco bag with the marked seasonal fluctuation that salinity presented. The high values in rainfall registered between June and September 2010 increased the flow rates of the rivers, especially Carinicuao and Cariaco, reflecting a decrease in salinity values in the rainy season. This temporal variation in salinity in the eastern end of the gulf coincided with that mentioned by Simpson and Griffiths (1967), Okuda et al. (1978) and more recently with Márquez et al. (2011), Martínez et al. (2011), Márquez-Rojas et al. (2017).

The seasonal distribution pattern observed in the total abundance of copepods, with the highest values recorded in the rainy season, was partly related to the behavior of Acartia tonsa, which was the most abundant species; this was also found in many other estuarine systems (Magalháes et al., 2009; Da Costa et al., 2011; Escamilla et al., 2011; Teiguel-C, 2015; RuízPineda et al., 2016). This planktonic calanoid copepod is a cosmopolitan species, dominant in many subtropical and temperate zones (Mauchline, 1998), it shows a high degree of tolerance to environmental changes, managing to normally withstand salinity intervals from 5 UPS (Cervetto et al., 1999) to 30 UPS (Calliari et al., 2006), exceptionally, samples have been found in systems with salinity from 0 UPS (Cronin et al., 1962) to 52 UPS (Rey et al., 1991).

The absence of a significant correlation between Acartia tonsa and salinity in the Gulf of Cariaco sack indicates that this species did not demonstrate a preference for estuarine waters. However, intraspecific tolerance to low and moderate salinities could explain the greater numerical amount of $A$. tonsa throughout the rainy season. For Temora turbinata, a species widely distributed in tropical and subtropical regions, a conspicuous member of superficial and near-surface mesozooplankton communities in estuaries and neritic and oceanic waters (Ara, 2002), the correlation between salinity and abundance was significant and positive. The 
la salinidad y la abundancia fue significativa y positiva. Las abundancias altas registradas en el período de sequía sugieren que la presencia de esta especie en el área estudiada, depende del aumento de la salinidad.

Ruíz-Pineda et al. (2016) mencionan que los géneros Acartia, Temora, Paracalanus y Labidocera son elementos faunísticos eurihalinos y euritérmicos, con afinidad estuarina. Por lo tanto, A. tonsa y T. turbinata ha sido reportada con gran éxito en medios estuarinos, en lagunas costeras con fuerte influencia marina y en zonas con influencia terrígena (Peck et al., 2015), ratificando una vez más la presencia de estas especies en el extremo oriental del golfo. Por su parte, P. quasimodo fue la tercera especie en abundancia en la presente investigación, con incrementos más altos en la época de lluvia; sin embargo, no se halló correlación con la salinidad. Los resultados en cuanto a la abundancia de esta especie coincidió con lo reportado por Villalba et al. (2017) en la laguna costera El Morro, isla de Margarita, Venezuela, donde la contabilizaron como la segunda especie más abundante durante el período de relajación. Por consiguiente, los copépodos A. tonsa, T. turbinata y P. quasimodo estuvieron presentes en ambos períodos estudiados y en elevadas abundancias; por lo tanto, se puede concluir que estas especies son residentes permanentes de este cuerpo de agua.

La gran variabilidad de las condiciones físicoquímicas en los estuarios, requiere que las especies que allí habitan tengan una mayor tolerancia a los cambios ambientales, lo que resulta en comunidades ecológicas menos diversas que en los ecosistemas acuáticos adyacentes, por lo que no solamente la tolerancia fisiológica de las especies es importante, sino también las estrategias de alimentación (Elliott y Whitfield, 2011; Márquez-Rojas et al., 2017). Por ejemplo, los copépodos típicamente estuarinos, A. tonsa y T. turbinata son conocidos por ser especies eurihalinas (Paffenhöfer, 1991; Ara, 2002; Hwang et al., 2006), incluso, la primera tiene un amplio intervalo de tolerancia (0-50 UPS) (Paffenhöfer, 1991; Rey et al., 1991; Martínez-Barragán et al., 2009), en tanto que $A$. tonsa tiene un espectro más amplio de alimentos con una mayor capacidad de seleccionar su presa entre la materia inorgánica, incluso debido a su capacidad omnívora, le permite ingerir grandes células de fitoplancton (que son comunes en medios con influencia de aguas continentales) y controlar a sus competidores potenciales, depredando a sus nauplios y copepoditos (Paffenhöfer, 1991), en contraste con T. turbinata que se alimenta principalmente de diatomeas high abundances recorded in the dry period suggest that the presence of this species in the studied area depends on the increase in salinity.

Ruíz-Pineda et al. (2016) mention that the genera Acartia, Temora, Paracalanus, and Labidocera are euryhaline and eurythermic fauna elements, with estuarine affinity. Therefore, A. tonsa and T. turbinata have been reported with great success in estuarine environments, in coastal lagoons with a strong marine influence and areas with a terrogenic influence (Peck et al., 2015), once again ratifying the presence of these species at the eastern end of the gulf. On the other hand, P. quasimodo was the third species in abundance in the present investigation, with higher increases in the rainy season; however, no correlation was found with salinity. The results regarding the abundance of this species coincided with that reported by Villalba et al. (2017) in the El Morro coastal lagoon, isla de Margarita, Venezuela, where it was counted as the second most abundant species during the relaxation period. Consequently, the copepods A. tonsa, T. turbinata, and P. quasimodo were present in both periods studied and in high abundances; therefore, it can be concluded that these species are permanent residents of this body of water.

The great variability of the physical-chemical conditions in estuaries requires that the species that inhabit them have a greater tolerance to environmental changes, resulting in less diverse ecological communities than in adjacent aquatic ecosystems, so not only the species physiological tolerance is important, but also feeding strategies (Elliott and Whitfield, 2011; Márquez-Rojas et $a l ., 2017)$. For example, typically estuarine copepods, $A$. tonsa, and T. turbinata are known to be euryhaline species (Paffenhöfer, 1991; Ara, 2002; Hwang et al., 2006), even the former has a wide tolerance range (0-50 UPS) (Paffenhöfer, 1991; Rey et al., 1991; Martínez-Barragán et al., 2009), while A. tonsa has a broader spectrum of food with a greater ability to select its prey among the material inorganic, even due to its omnivorous capacity, allows it to ingest large phytoplankton cells (which are common in environments influenced by inland waters) and control its potential competitors, preying on its nauplii and copepodites (Paffenhöfer, 1991), in contrast to $T$. turbinata that feeds mainly on diatoms with frustules (Eskinazi-Sant'anna, 2000). This $A$. tonsa feeding strategy is an advantage in the eastern end of the Gulf of Cariaco, due to the influence of inland waters, it is an area with high turbidity, high content of suspended matter, high 
con frústulas (Eskinazi-Sant'anna, 2000). Esta estrategia de alimentación de $A$. tonsa es una ventaja en el extremo oriental del golfo de Cariaco, debido a la influencia de las aguas continentales, es una zona con alta turbidez, alto contenido de materia en suspensión, concentraciones altas de nutrientes principalmente de origen terrestre (Márquez et al., 2011; Martínez et al., 2011). Además, esta premisa quedó confirmada con la relación directa de $A$. tonsa con la salinidad y la clorofila $a$ en el ACC, durante la época de lluvias. De igual manera, Camatti et al. (2019) corroboraron que la distribución estacional de $A$. tonsa en la laguna de Venecia, mar Mediterráneo, estaba asociada positiva y significativamente con la temperatura, el fitoplancton, la clorofila $a$ y el gradiente de salinidad, confirmando que es una especie tolerante oportunista que puede tomar provecho de los ecosistemas eutróficos.

En el extremo oriental del golfo de Cariaco, la diversidad no mostró diferencias estacionales significativas; no obstante, la comunidad de copépodos presentó valores un poco más altos en lluvia. Este ligero aumento durante los meses de lluvias probablemente estuvo relacionado con la mayor riqueza de especies registradas en este período, a pesar que durante estos meses hubo una mayor contribución de $A$. tonsa en la abundancia total de los copépodos. En este sentido, la abundancia de $A$. tonsa se incrementó con el descenso de la salinidad, debido al incremento de la descarga de los ríos Carinicuao y Cariaco que descargan justamente en la zona de estudio; sin embargo, no se encontró una correlación significativa entre esta especie y la salinidad, donde se pueda deducir que la salinidad la afecta directamente. Numerosos estudios en estuarios han descrito la reducción en la diversidad de los copépodos durante la estación lluviosa (Mwaluma et al., 2003; Osore et al., 2004; Magalháes et al., 2015); sin embargo, se verificó una tendencia opuesta en el saco del golfo de Cariaco. Los valores bajos de diversidad de copépodos obtenidos en el sector oriental del golfo de Cariaco coinciden con lo encontrado por Magalháes et al. (2009) en el estuario de Curuçá, Brasil (0,5-2,7 bits/ind $\left.{ }^{-1}\right)$ y por Ruíz-Pineda et al. (2016) en el sistema estuarino de la bahía de Chetumal, México (1,1-1,8 bits/ind $\left.d^{-1}\right)$.

La equidad a diferencia de la diversidad, si presentó diferencia estacional; no obstante, siguió el mismo patrón de la diversidad, con valores más altos en lluvia. Esta situación coincidió con un incremento en la frecuencia y abundancia de los géneros de hábitos estuarinos Labidocera, Pseudodiaptomus y Saphirella y podría ser el resultado de concentrations of nutrients mainly of origin terrestrial (Márquez et al., 2011; Martínez et al., 2011). Furthermore, this premise was confirmed with the direct relationship of $A$. tons $a$ with salinity and chlorophyll- $a$ in the ACC, during the rainy season. Similarly, Camatti et al. (2019) corroborated that the seasonal distribution of $A$. tonsa in the Venice lagoon, Mediterranean Sea, was positively and significantly associated with temperature, phytoplankton, chlorophyll- $a$ and the salinity gradient, confirming that it is an opportunistic tolerant species that can take advantage of eutrophic ecosystems.

At the eastern end of the Gulf of Cariaco, diversity did not show significant seasonal differences; however, the copepod community showed slightly higher values in rain. This slight increase during the rainy months was probably related to the greater species richness recorded in this period, although during these months there was a greater contribution of $A$. tonsa in the total abundance of copepods. In this sense, the abundance of $A$. tons $a$ increased with the decrease in salinity, due to the increase in the discharge of the Carinicuao and Cariaco rivers that discharge precisely in the study area; however, no significant correlation was found between this species and salinity, where it can be deduced that salinity affects it directly. Numerous studies in estuaries have described the reduction in the diversity of copepods during the rainy season (Mwaluma et al., 2003; Osore et al., 2004; Magalháes et al., 2015); however, an opposite trend was verified in the Gulf of Cariaco sack. The low values of copepod diversity obtained in the eastern sector of the Gulf of Cariaco coincide with what was found by Magalháes et al. (2009) in the Curuçá estuary, Brazil (0.5-2.7 bits/ind $\left.{ }^{-1}\right)$ and by Ruíz-Pineda et al. (2016) in the Chetumal Bay estuarine system, Mexico (1.1-1.8 bits/ind $\left.{ }^{-1}\right)$.

Equity unlike diversity, if it presented seasonal differences; however, it followed the same pattern of diversity, with higher values in rainfall. This situation coincided with an increase in the frequency and abundance of the genus of estuarine habits Labidocera, Pseudodiaptomus, and Saphirella and could be the result of a phenomenon of "drift" of zooplankton from the adjacent Caribbean Sea. According to Bradford-Grieve et al. (1999) and Ordóñez-López and Ornelas-Roa (2003), these genera with their estuarine-coastal condition are frequent in the coastal zooplankton and their presence in the Gulf of Cariaco sac indicates fauna exchange between estuarine and marine environments. The slight increase 
un fenómeno de "deriva" del zooplancton proveniente del mar Caribe adyacente. De acuerdo con Bradford-Grieve et al. (1999) y Ordóñez-López y Ornelas-Roa (2003) estos géneros con su condición estuarina-costera son frecuentes en el zooplancton costero y su presencia en el saco del golfo de Cariaco indica intercambio de fauna entre los ambientes estuarinos y marinos. El ligero aumento de los índices ecológicos del zooplancton en la época de lluvias apoya esta hipótesis.

Por consiguiente, se evidenció que durante la época de lluvias se incrementó significativamente el número de individuos y la ocurrencia de ciertas especies. Esto también fue registrado en varios sistemas estuarinos tropicales, registrando densidades altas durante la temporada de lluvias, como por ejemplo el trabajo de Sterza y Fernandes (2006) en la bahía Vitória, sureste de Brasil, el de Garboza da Costa et al. (2008) y Da Costa et al. (2011) en el estuario Taperaçu y Caeté, respectivamente, norte de Brasil. De hecho, estudios sobre la comunidad de copépodos realizados en zonas estuarinas de regiones tropicales y subtropicales, mencionados anteriormente, coinciden en que la salinidad es el factor determinante en la distribución espacial de algunas especies mientras que factores como la lluvia, la temperatura y el oxígeno disuelto pueden determinan la distribución temporal (Villate et al., 2017).

Diversos trabajos han discutido largamente la relación de los zooplanctéres y el ambiente estuarino, sin embargo es evidente que la abundancia de las poblaciones cambian (Day et al., 1989; Ordóñez-López y Ornelas-Roa, 2003). En este sentido, el gradiente halino, la temperatura y la descarga de agua dulce no fueron los únicos factores que determinaron los patrones de distribución de los copépodos planctónicos, es probable que factores biológicos como el flujo de estadios tempranos (naupliares o copepoditos), la competencia y la depredación tengan un papel importante en la variación de la abundancia de los organismos para las poblaciones de copépodos del saco del golfo de Cariaco. Estas presunciones también son apoyadas por Chaalali et al. (2013) y David et al. (2016), quienes mencionan que la coexistencia y dominancia de pocas especies en el mismo sistema podría ser explicado por las segregaciones espaciales y temporales causadas por las diferencias en las estrategias reproductivas y la dieta.

Los cambios mensuales y estacionales en el balance de la abundancia relativa de las principales especies de copépodos como se visualiza en las curvas de K-dominancia, influyeron directamente en el comportamiento de los índices in ecological indices of zooplankton in the rainy season supports this hypothesis.

Consequently, it was evident that during the rainy season the number of individuals and the occurrence of certain species increased significantly. This was also recorded in several tropical estuarine systems, registering high densities during the rainy season, such as the work of Sterza and Fernandes (2006) in Vitória Bay, southeast Brazil, that of Garboza da Costa et al. (2008) and Da Costa et al. (2011) in the Taperaçu and Caeté estuaries, respectively, northern Brazil. Studies on the copepod community carried out in estuarine areas of tropical and subtropical regions, mentioned above, agree that salinity is the determining factor in the spatial distribution of some species, while factors such as rainfall, temperature, and dissolved oxygen can determine the temporal distribution (Villate et al., 2017).

Various studies have long discussed the relationship between zooplankton and the estuarine environment, however, it is evident that the abundance of population changes (Day et al., 1989; Ordóñez-López and Ornelas-Roa, 2003). In this sense, the haline gradient, the temperature, and the freshwater discharge were not the only factors that determined the distribution patterns of the planktonic copepods, it is probable that biological factors such as the flow of early stages (naupliar or copepodites), the Competition and predation play an important role in the variation of the abundance of organisms for the copepod populations of the Gulf of Cariaco sack. These assumptions are also supported by Chaalali et al. (2013) and David et al. (2016), who mentioned that the coexistence and dominance of few species in the same system could be explained by spatial and temporal segregations caused by differences in reproductive strategies and diet.

The monthly and seasonal changes in the balance of the relative abundance of the main copepod species as visualized in the $\mathrm{K}$-dominance curves directly influenced the behavior of the ecological indices. In particular, the changes in the contribution of $A$. tonsa in the periods studied were responsible for the changes in these indices.

The present study has not shown, in the eastern sector of the Gulf of Cariaco, a clear seasonal pattern in the abundance, diversity, and uniformity of the copepod community and the salinity values, which has also been visualized in the cluster and multidimensional scaling analysis (MDS). Therefore, the studied area can be 
ecológicos. En particular, los cambios en la contribución de A. tonsa en los períodos estudiados fueron responsables de las alteraciones en estos índices.

El presente estudio no ha mostrado, en el sector oriental del golfo de Cariaco, un claro patrón estacional en la abundancia, diversidad y la uniformidad de la comunidad de copépodos y los valores de salinidad, lo cual también se ha visualizado en el cluster y en el análisis de escalamiento multidimensional(MDS). Por lo tanto, el área estudiada puede considerarse espacialmente uniforme y estacionalmente homogéneo en relación a estos parámetros. Sin embargo, hay que mencionar que la comunidad de copépodos registró una mayor abundancia y riqueza de especies en el período de lluvias. Por lo tanto, investigaciones nuevas sobre la biomasa y estructura comunitaria de los copépodos en el saco del golfo de Cariaco, deben ser evaluadas para proporcionar una mejor comprensión de la estructura trófica de este ecosistema.

En definitiva, se sabe que los copépodos son particularmente sensibles a los cambios ambientales, ya sea como resultado de las condiciones ambientales naturales o por acción antropogénica (Li et al., 2000; Camatti et al., 2019). La interacción entre la actividad antropógena, el cambio climático y las comunidades planctónicas, centrándose en los cambios sistemáticos en la estructura comunitaria, en la abundancia y la distribución en las últimas décadas, es un tema global clave, así como los potenciales impactos socioeconómicos de estos cambios (Hays et al., 2005). En ambientes con constantes cambios, como el extremo oriental del golfo de Cariaco, por la influencia de la surgencia costera, la descarga de las aguas servidas y la sobrepesca, el monitoreo de la dinámica ecológica de las especies es de suma importancia. En particular, deben considerarse las modificaciones continuas de los ensamblajes de copépodos en respuesta a factores estresantes antropógenicos y ambientales. El monitoreo continuo del plancton actuará como investigación centinela para identificar futuros cambios en este complejo ecosistema fuertemente impactado y su red alimentaria relacionada que proporciona sustento a especies como la sardina (Sardinella aurita), el principal recurso pesquero en el golfo (Quintero et al., 2002).

\section{PARTICIPACIÓN DE AUTORES}

MRB Toma de la muestra, análisis de datos y escritura del documento, TL análisis de datos estadísticos y escritura del manuscrito y ZRE concepción, diseño y escritura del documento. considered spatially uniform and seasonally homogeneous with these parameters. However, it should be mentioned that the copepod community registered a greater abundance and species richness in the rainy season. Therefore, new research on the biomass and community structure of copepods in the Gulf of Cariaco sack should be evaluated to provide a better understanding of the trophic structure of this ecosystem.

Ultimately, copepods are known to be particularly sensitive to environmental changes, either as a result of natural environmental conditions or by anthropogenic action (Li et al., 2000; Camatti et al., 2019). The interaction between anthropogenic activity, climate change, and planktonic communities, focusing on systematic changes in community structure, abundance, and distribution in recent decades, is a key global issue, as well as the potential socio-economic impacts of these changes (Hays et al., 2005). In environments with constant changes, such as the eastern end of the Gulf of Cariaco, due to the influence of coastal upwelling, sewage discharge, and overfishing, monitoring the ecological dynamics of the species is of utmost importance. In particular, continuous modifications of copepod assemblies in response to anthropogenic and environmental stressors should be considered. Continuous monitoring of plankton will act as sentinel research to identify future changes in this highly impacted complex ecosystem and its related food web that provides sustenance for species such as sardines (Sardinella aurita), the main fishing resource in the gulf (Quintero et al., 2002).

\section{AUTHORS PARTICIPATION}

MRB Sample taking, data analysis, and writing of the document, TL analysis of statistical data and writing of the manuscript and ZRE conception, design, and writing of the document.

\section{ACKNOWLEDGEMENT}

The authors thank the support staff and technicians of the Venezuelan Oceanographic Institute who participated in the field and laboratory work. The first author thanks the Research Council of the Universidad de Oriente, for the financial support through the project entitled Planktonic study in the Gulf of Cariaco sack (CI2-030601-1411/08). 


\section{AGRADECIMIENTOS}

Los autores agradecen al personal de apoyo y técnicos del Instituto Oceanográfico de Venezuela que participaron en el trabajo de campo y laboratorio. La primera autora agradece al Consejo de Investigación de la Universidad de Oriente, por el apoyo económico a través del proyecto intitulado Estudio planctónico en el saco del golfo de Cariaco (CI-2-030601-1411/08).

\section{BIBLIOGRAFÍA/LITERATURE CITED}

Ara, K. 2002. Variabilidad temporal y producción de Temoraturbinata (Copepoda: Calanoida) en el complejo estuarino-lagunar de Cananéia, São Paulo, Brasil. Sci. Mar., 66(4): 399-406. doi:10.3989/scimar.2002.66n4399

Beisner, B.E. 2001. Plankton community structure in fluctuating environments and the role of productivity. Oikos. 95: 496-510. doi:10.1034/j.16000706.2001.950315.x.

Bonilla, J., A. Márquez y B. Gamboa. 1985. Características geoquímicas en núcleos de sedimentos de la región nororiental y río Orinoco, Venezuela. Bol. Inst. Oceanogr. Venez., 24(1- 2): 43-61.

Bradford-Grieve, J., E.L. Markhaseva, C.E. Rocha y B. Abiahy. 1999. Copepoda. 869-1098. En: Boltovskoy, D. (Ed.). South Atlantic Zooplankton, Netherlands. Backhuys. 1706 p.

Branco, C.W., B. Kozlowsky-Suzuki and F.A. Esteves. 2007. Environmental changes and zooplankton temporal and spatialvariation in a disturbed Brazilian coastal lagoon. Braz. J. Biol., 67: 251-262. doi:10.1590/S1519-69842007000200010.

Briceño, H., R. Buonocore, C. Sangronis, L. García, J. Rojas, J. Chirinos, A. González y C. López. 2009. Composición y abundancia del plancton de la Costa noreste de la bahía El Tablazo, Sistema de Maracaibo, Venezuela. Bol. Centro Invest. Bio., 43(4): 463-485.

Calliari, D., C.M. Andersen, P. Thor, E. Gorokhova and P. Tiselius. 2006. Salinity modulates the energy balance and reproductive success of co-occuring copepods Acartia tonsa and Acartia clause in different ways. Mar. Ecol. Prog. Ser., 312: 177-188.

Camatti, E., M. Pansera and A. Bergamasco. 2019. The copepod Acartia tonsa dana in a microtidal Mediterranean lagoon: history of a successful invasion. Water, 11(6): 1200. doi:10.3390/w11061200.

Campos-Hernández, A y E. Suárez-Morales. 1994. Copépodos pelágicos del golfo de México y mar Caribe. 1. Biología y Sistemática. Centro de Investigaciones de Quintana Roo. Conacyt. México. 398 p.

Caraballo, M. 1982. El golfo de Cariaco. Parte I. Morfología y batimetría submarina. Estructuras y tectonismo reciente. Bol. Inst. Oceanogr., 21(1 y 2): 13-35.

Cervetto, G., R. Gaudy and M. Pagano. 1999. Influence of salinity on the distribution of Acartia tonsa (Copepoda, Calanoida). J. Exp. Mar. Biol. Ecol., 239(1): 33-45. doi:10.1016/S0022-0981(99)00023-4.

Chaalali, A., G. Beaugrand, V. Raybaud, E. Goberville, V. David, P. Boëty and B. Sautour. 2013. Climatic facilitation of the colonization of an estuary by Acartia tonsa. PLoS ONE 8: e74531. doi:10.1371/journal.pone.0074531.

Clarke, K and R. Gorley. 2006. PRIMER v5 (and v6): User manual/tutorial. Plymouth: PRIMER-E.

Cochran, W.G. 1977. Sampling Techniques. (3rd edition). Wiley, New York.

Cronin L.E., J.D. Daiber and E.M. Hulbert. 1962. Quantitative seasonal aspects of zooplankton in the Delaware River Estuary. Chesapeake Sci., 3: 63-93.

Da Costa, R.M., P. Atique, K.G. Da Costa and L.C. Pereira. 2011. Seasonal and spatial variation in hydrological parameters and microzooplankton communities in an Amazonian estuary. J. Coastal. Res., (64): 1477-1481.

David, V., J. Selleslagh, A. Nowaczyk, S. Dubois, G. Bachelet, H. Blanchet, B. Gouillieux, N. Lavesque, M. Leconte, N. Savoye, B. Sautour and J. Lobry. 2016. Estuarine habitats structure zooplankton communities: Implications for the pelagic trophic pathways. Estuar. Coast. Shelf. S., 179: 99-111. doi:10.1016/j. ecss.2016.01.022.

Day. J.W., C.A. Hall, W.M. Kemp and A. Yanez-Arancibia. 1989. Estuarine ecology. John Wiley and Sons, New York.

Elliott, M. and A.K. Whitfield. 2011. Challenging paradigms in estuarine ecology and management. Estuar. Coast. Shelf. S., 94(4): 306-314. doi:10.1016/j. ecss.2011.06.016.

Escamilla, J.B., U. Ordóñez-López U y E. Suárez-Morales. 2011. Variabilidad espacial y estacional de Acartia (Copepoda) en una laguna costera del sur del golfo de México. Rev. Biol. Mar. Oceanogr., 46: 379-390. doi:10.4067/S0718-19572011000300008.

Eskinazi-Sant'anna, E.M. 2000. Estudo da dieta natural de Parvocalanus crassirostris, Paracalanus quasimodo, Temora stylifera e Temora turbinata (Copepoda, Calanoida), no Canal de São Sebastião (SP, Brasil). São Paulo, Tese (Doutorado em Oceanografia). Universidade de São Paulo. 
Garboza da Costa, I.K., L.C. Carneiro y M. Da Costa. 2008. Short and long-term temporal variation of the zooplankton in a tropical estuary (Amazon region, Brazil). Bol. Mus. Para. Emílio Goeldi. Cienc. Nat., Belém. 3(2): 127-141.

Gómez, A. 1983. Pigmentos clorofílicos, producción primaria y abundancia planctónica en el canal de entrada a la laguna de La Restinga, Venezuela. Bol. Inst. Oceanogr. Venez., 22(1- 2): 43-63.

Hays, G.C., A.J. Richardson and C. Robinson. 2005. Climate change and marine plankton. Trends Ecol. Evol., 20: 337-344. doi:10.1016/j.tree.2005.03.004.

Hwang, J.S., S. Souissi, L.C. Tseng, L. Seuront, F. Schmitt and L.S. Fang. 2006. A 5-year study of the influence of the northeast and southwest monsoons on copepod assemblages in the boundary coastal waters between the East China Sea and the Taiwan Strait. J. Plank. Res., 28(10): 943-958. doi:10.1093/ plankt/fb1031.

Infante, J. y L. Urosa. 1986. Distribución vertical de copépodos en aguas deficientes de oxígeno. Bol. Inst. Oceanogr. Venez., 25(1-2): 175-194.

Kiørboe, T. 2011. What makes pelagic copepods so successful? J. Plank. Res., 33(5): 677-685

Krebs, C.J. 1999. Ecological methodology. Second edition. New York: Addison-Wesley. Educational Publishers, Inc., Menlo Park. 620 p.

Lambshead, P.J., H.M. Platt and K.M. Shaw. 1983. The detection of differences among assemblages of marine benthic species based on an assessment of dominance and diversity. J. Nat. Hist., 17: 859-874.

Legaré, E.H. 1961. Estudios preliminares del zooplancton en la región de Cariaco. Bol. Inst. Oceanogr. Venez., 1(1): 191-218.

Legendre, P. and L. Legendre. 1998. Numerical ecology. 2nd English Edition, Elsevier Science, Amsterdam. 852 p.

Li, M., A. Gargett and K. Denman. 2000. What determines seasonal and interannual variability of phytoplankton and zooplankton in strongly estuarine systems? Application to the semi enclosed estuary of Strait of Georgia and Juan de Fuca Strait. Estuar. Coast. Shelf. Sci., 50: 467-488.

López-Monroy, F. y L. Tróccoli. 2015. Aproximación sobre la climatología de la isla de Margarita y su importancia en los procesos oceánicos. Saber, 26(4): 467-471.

López-Peralta, R.H. y L.H. Mojica-López. 2015. Influencia abiótica sobre algunos géneros de copépodos (Crustacea) epipelágicos en el pacífico colombiano. Septiembre de 2002. Revista Fac. Ciencias B. Universidad Militar Nueva Granada, 11(1): 20-33.

Lorenzoni, L., G.T. Taylor, C. Benítez, D.A. Hansell, E. Montes, R. Masserini, K. Fanning, R. Varela, Y. Astor, L. Guzmán and F. Muller-Karger. 2013. Spatial and seasonal variability of dissolved organic matter in the Cariaco Basin. J. Geophys. Res-Biogeo., 118:951-962. doi.org/10.1002/jgrg.20075.

Magalhães, A., L.C. Pereira and R.M. Costa. 2015. Relationships between copepod community structure, rainfall regimes, and hydrological variables in a tropical mangrove estuary (Amazon coast, Brazil). Helgol. Mar. Res., 69(1): 123-136. https://doi.org/10.1007/s10152-014-0421-4.

Magalhães, A., N. Leite, J.G. Silva, L.C. Pereira and R.M. Da Costa. 2009. Seasonal variation in the copepod community structure from a tropical Amazon estuary, Northern Brazil. An. Acad. Bras. Ciênc., 81(2): 187-197.

Marques, S.C., U.M. Azeiteiro, J.C. Marques, J. Neto and M.A. Pardal. 2006. Zooplankton and ichthyoplankton communities in a temperate estuary: spatial and temporal patterns. J. Plank. Res., 28(3): 297-312. doi:10.1093/plankt/fbi126.

Márquez, A., W. Senior, A. Benítez, I. Fermín, G. Martínez, A. González, J. Castañeda, L. Alcalá y R. De la Cruz. 2011. Sector oriental del golfo de Cariaco, Venezuela. Una descripción de su dinámica hidroquímica, procesos y del rol de la surgencia costera estacional. Bol. Inst. Oceanogr. Venez., 50(2): 255-272.

Márquez-Rojas, B., B. Marín, E. Zoppi y C. Moreno. 2006. Zooplancton del golfo de Cariaco. Bol. Inst. Oceanogr. Venez., 45(1): 61-78.

Márquez-Rojas, B., E. Zoppi de Roa, L. Tróccoli y E. Montiel. 2017. Cambios estructurales del mesozooplancton en relación a las condiciones hidrográficas en el golfo de Cariaco, Venezuela. Biota Colombiana 18(1): 148-171. DOI: 10.21068/c2017.v18n01a9.

Márquez-Rojas, B., L. Tróccoli, L. Marcano, J. Morales, T. Allen, B. Marín y R. Díaz. 2011. Estructura comunitaria del zooplancton en dos localidades del golfo de Cariaco, Venezuela. Bol. Inst. Oceanogr. Venez., 45(1): 61-78.

Márquez-Rojas, B., O. Díaz-Díaz, L. Tróccoli, J. Morales y L.M. Marcano. 2014a. Corycaeidae Dana, 1852 (Copepoda: Poecilostomatoida) del golfo de Cariaco, Venezuela. MES., 9(3): 1-18.

Márquez-Rojas, B., O. Díaz-Díaz, L. Tróccoli, J. Morales y L.M. Marcano. 2014b. Distribución espacial y abundancia de la Familia Corycaeidae Dana, 1852 (Copepoda: Poecilostomatoida) en el golfo de Cariaco, Venezuela. Bol. Inst. Oceanogr. Venez., 53(2): 221-233.

Martínez, G., D. Hernández, A. Quintero, A. Márquez, W. Senior y A. González. 2011. Estudio fisicoquímico de las aguas del sector oriental del golfo de Cariaco, Venezuela. Bol. Inst. Oceanogr. Venez., 50(2): 273-287.

Martínez-Barragán, M., J. Medina-Calderón, A. Franco-Herrera y A. Santos-Martínez. 2009. La comunidad de copépodos (Crustacea) en las islas de Providencia y Santa Catalina (Caribe colombiano) durante el período lluvioso de 2005. Bol. Invest. Mar. Cost., 38(1): 85-103.

Mauchline, J. 1998. The Biology of Calanoid copepods. Advaces in Marine Biology. V. 33, Academic Press, New York. 710p.

Mclusky, D.S. and M. Elliott. 2004. The estuarine ecosystem: ecology, threats and management. Oxford University Press, New York. 216 p.

Mwaluma, J., M. Osore, J. Kamau and P. Wawiye. 2003. Composition, abundance and seasonality of zooplankton in Mida Creek, Kenya. Western Indian Ocean J. Mar. Sci., 2(2): 147-155. doi:10.4314/wiojms.v2i2.28440. 
Neumann-Leitão, S., P.A. Melo, R. Schwamborn, X.F. Diaz, L.G. Figueiredo, A.P. Silva and M. Araújo. 2018. Zooplankton from a reef system under the influence of the Amazon River plume. Front. Microbiol., 9: 355-361. doi:10.3389/fmicb.2018.00355.

Okuda, T., J. Bonilla, J. Benítez, A. García y G. Cedeño. 1978. Características hidrográficas del golfo de Cariaco, Venezuela. Bol. Inst. Oceanogr. Venez., 17(12): $69-88$.

Ordóñez-López, U. y M. Ornelas-Roa. 2003. Variación de la comunidad de copépodos pláncticos en el gradiente estuarino-costero de Celestún, Yucatán, México. Hidrobiológica, 13: 231-238.

Osore, M.K., J.M. Mwaluma, F. Fiers and M.H. Daro. 2004. Zooplankton composition and abundance in Mida Creek, Kenya. Zool. Stud., 43(2): 415-424.

Paffenhöfer, G. 1991. Some characteristics of abundant subtropical copepods in estuarine, shelf and oceanic waters. Proc. 4th International Conference on Copepoda. Bull. Plank. Soc. Jpn. Spec., 1: 201-216.

Peck, N., J. Peters, R. Diekmann, S. Laakmann and J. Renz. 2015. Interactive effects of temperature and salinity on populations of the calanoid copepod Acartia tonsa. J. Plank. Res., 37(1): 197-210. doi:10.1093/plankt/fbu093.

Quintero, A., G. Terejova, G. Vicent, A. Padrón y J. Bonilla J. 2002. Los pescadores del golfo de Cariaco. Interciencia, 27(6): $286-292$.

Razouls, C., F. De Bovée, J. Kouwenberg y N. Desreumaux. 2005-2019. Diversity and geographic distribution of marine planktonic copepods. http://copepodes. obs-banyuls.fr/en. 19/07/2019.

Rey, J.R., T. Kain, R. Crossman, M. Peterson, J. Shaffer y F. Vose. 1991. Zooplankton of impounded marshes and shallow areas of a subtropical lagoon. Florida Scientist., 54(3-4): 191-203.

Rice, E., H.G. Dam y G. Stewart. 2015. Impact of climate change on estuarine zooplankton: surface water warming in long island sound is associated with changes in copepod size and community structure. Estuaries and Coasts, 38:13-23. doi:10.1007/s12237-014-9770-0.

Rose, T.H., J.R. Tweedley, R.M. Warwick y I.C. Potter. 2019. Zooplankton dynamics in a highly eutrophic microtidal estuary. Mar. Pollut. Bull., $142: 433-451$. https://doi.org/10.1016/j.marpolbul.2019.03.047.

Ruíz-Pineda, C., E. Suárez-Morales y R. Gasca. 2016. Copépodos planctónicos de la bahía de Chetumal, Caribe mexicano: variaciones estacionales durante un ciclo anual. Rev. Biol. Mar. Oceanogr., 51(2): 301-316. doi: 10.4067/S0718-19572016000200008.

Seda, J. and M. Devetter. 2000. Zooplankton community structure along a trophic gradient in a canyon-shaped dam reservoir. J. Plank. Res., 22(10): 1829-1840. doi:10.1093/plankt/22.10.1829.

Sheskin, D. 2004. Parametric and nonparametric statistical procedures. 4ed. Boca Ratón, Florida: Chapman \& Hall. 585 p.

Simpson, J. y R. Griffith. 1967. La distribución de densidad pesquera en el golfo de Cariaco, Venezuela. Serie: Recursos y explotación pesqueros, 1(8): 305-320.

Sterza, J.M. and L. Fernandes. 2006. Zooplankton community of the Vitória Bay estuarine system (Southeastern Brazil). Characterization during a three-year study. Braz. J. Oceanogr., 95-105. doi: 10.1590/S1679-87592006000200001.

Strickland, J.D. and T.R. Parsons. 1972. A practical handbook of seawater analysis. 2ed. J. Fish. Res. Board.Can. Bulle. 167 p.

Suárez-Morales, E. 1995. Clave ilustrada para la identificación de los copépodos pláncticos de la bahía de Chetumal. Ava. Cient., 12 : 16-24.

Teiguel-C, N.K. 2015. Efecto del estrés halino en la tasa de crecimiento y Reproducción de Acartia tonsa en el estuario del río Valdivia. Universidad Austral de Chile. Tesis de maestría. Chile. 177 p.

Ter Braak, C.J. and P.E. Verdonschot. 1995. Canonical correspondence analysis and related multivariate methods in aquatic ecology. Aquat. Sci., 57(3): 255-289.

Vásquez-Yeomans, L., I. Castellanos, E. Suárez-Morales y R. Gasca. 2012. Variación espacio-temporal de la biomasa de zooplancton en un sistema estuarino del Caribe Occidental durante dos ciclos anuales. Rev. Biol. Mar. Oceanogr., 47(2): 213-225. doi.org/10.4067/S0718-19572012000200005.

Villalba, W., Márquez-Rojas, B., L. Tróccoli, M. Alzolar y J. López. 2017. Composición y abundancia del zooplancton en la laguna El Morro, isla de Margarita, Venezuela. Rev. Peru. Biol., 24(4): 343-356. doi: http://dx. doi.org/10.15381/rpb.v24i4.14062.

Villate, F., A. Iriarte, I. Uriarte and I. Sánchez. 2017. Seasonal and interannual variability of mesozooplankton in two contrasting estuaries of the Bay of Biscay: Relationship to environmental factors. J. Sea Res., 130: 189-203. doi:10.1016/j.seares.2017.05.002.

Vukanic, V., N. Glisovic, T. Jaksic, N. Zivic y D. Vukanic. 2018. Seasonal abundance of copepods in the Bojana Estuary (Southeastern Adriatic Sea). Fresen Environ Bull., 27: 7209-7221.

Zar, J.H. 1999. Biostatistical Analysis, 4th ed., Prentice Hall, Englewoods Cliff, New Jersey. 699 p.

Zoppi, E. 1961. Distribución vertical del zooplancton en el golfo y extremo este de la fosa de Cariaco. Bol. Inst. Oceanogr. Venez., 1(1): $219-248$.

Zoppi, E. 1974. Comparación de algunas características del plancton entre las lagunas costeras de Tacarigua y Unare, Venezuela. Bol. Inst. Oceanogr. Venez., 13(1-2): 129-146. 